\title{
Luminescent Supramolecular Nano- or Microstructures Formed in Aqueous Media by Amphiphile-Noble Metal Complexes
}

\author{
Carmen Cretu $\mathbb{D}^{1},{ }^{1}$ Loredana Maiuolo $\mathbb{D}^{2},{ }^{2}$ Domenico Lombardo $\mathbb{D}^{3}{ }^{3}$ Elisabeta I. Szerb $\left.\mathbb{D}\right)^{1}$ \\ and Pietro Calandra iD ${ }^{4}$ \\ 1 "Coriolan Drăgulescu" Institute of Chemistry, Romanian Academy, 300223 Timisoara, Romania \\ ${ }^{2}$ Dipartimento di Chimica e Tecnologie Chimiche, Università della Calabria, 87036 Arcavacata di Rende (CS), Italy \\ ${ }^{3}$ CNR-IPCF, Consiglio Nazionale delle Ricerche, Istituto per i Processi Chimico-Fisici, 98158 Messina, Italy \\ ${ }^{4}$ CNR-ISMN, Consiglio Nazionale delle Ricerche, Istituto per lo Studio dei Materiali Nanostrutturati, 00015, Monterotondo Stazione, \\ Rome, Italy
}

Correspondence should be addressed to Elisabeta I. Szerb; szella73@gmail.com and Pietro Calandra; pietro.calandra@cnr.it

Received 30 June 2020; Revised 18 August 2020; Accepted 18 September 2020; Published 13 October 2020

Academic Editor: Elisabetta Comini

Copyright (c) 2020 Carmen Cretu et al. This is an open access article distributed under the Creative Commons Attribution License, which permits unrestricted use, distribution, and reproduction in any medium, provided the original work is properly cited.

\begin{abstract}
The involvement of metal ions within the self-assembly spontaneously occurring in surfactant-based systems gives additional and interesting features. The electronic states of the metal, together with the bonds that can be established with the organic amphiphilic counterpart, are the factors triggering new photophysical properties. Moreover, the availability of stimuli-responsive supramolecular amphiphile assemblies, able to disassemble in a back-process, provides reversible switching particularly useful in novel approaches and applications giving rise to truly smart materials. In particular, small amphiphiles with an inner distribution, within their molecular architecture, of various polar and apolar functional groups, can give a wide variety of interactions and therefore enriched self-assemblies. If it is joined with the opportune presence and localization of noble metals, whose chemical and photophysical properties are undiscussed, then very interesting materials can be obtained. In this minireview, the basic concepts on self-assembly of small amphiphilic molecules with noble metals are shown with particular reference to the photophysical properties aiming at furnishing to the reader a panoramic view of these exciting problematics. In this respect, the following will be shown: (i) the principles of self-assembly of amphiphiles that involve noble metals, (ii) examples of amphiphiles and amphiphile-noble metal systems as representatives of systems with enhanced photophysical properties, and (iii) final comments and perspectives with some examples of modern applications.
\end{abstract}

\section{Introduction}

Modern technology applications have ever-increasing demand of hybrid nanomaterials based on metal complexes not only because of their small size but also thanks to the presence of exotic properties arising from the combination of molecular properties and novel and/or synergistic emergent ones. Electronic states and optical properties, indeed, are the consequence of the various interactions involved and of the specific self-assembly, which can be sensitive to various external stimuli $[1,2]$. An efficient protocol for the piloted design of nanomaterials is in general to exploit the natural tendency of intermolecular self-assembly triggered by the various polar, apolar, H-bonds, ionic, atomic, etc. interactions.

Self-assembly in amphiphiles is particularly interesting: they in fact simultaneously possess both polar and apolar moieties within their molecular architecture [3, 4]. So, various and different possible intermolecular interactions can be simultaneously established: polar-polar, polar-apolar, and apolar-apolar interactions, as well as steric hindrance and eventual directional $\mathrm{H}$-bonds. The overall result is the formation of supramolecular structures (micelles, vesicles, nanotubes, nanofibres, or lamellae) with peculiar structures 
and properties mimicking biological systems $[5,6]$ and of added value in bionanotechnology, drug and gene delivery, and materials science [7].

The presence of coordination bonds with metal atoms further enriches the scenario of self-assembly and the relative applications. In this ambit, noble metals are the best candidates since their chemical and optical properties are peculiar and well studied [8]. The high number of electrons in metals gives specific properties and functions [9]. Usually, the properties are photophysical properties, which, if present in stimuli-responsive amphiphile-metal assemblies, can give rise to smart materials. In such materials, the assembly/disassembly (forward and back-processes) can be used for molecular optical switches.

Here, we want to give a modern vision of the topic, introducing the simultaneous consideration of two elements:

(1) A schematic and general vision based of the physics of complex systems. The possibility of a metal to bind molecular functional groups offers new possibilities in their intermolecular assembly. Usually, organic materials and inorganic ones are dealt with different disciplines (organic and inorganic chemistry, respectively) since they have different types of interactions. Despite facing different aspects, chemists are perfectly aware that two independently stable compounds can combine to form a new chemical compound with properties quite different from those of the constituents; this is usually seen as a "reaction" giving a change of the system. This vision is correct, of course, but we want here to present an alternative vision borrowing the concepts from the physics of complex systems: the different types of interactions are the perfect prerequisites for the rising of the socalled emerging properties, i.e., those properties that cannot be traced back to the single constituents (metals or organic molecules) but only to their collective/cooperative behaviour. Their evaluation, rationalization, and study involve the facing of manybody systems characterized by many bonds among them and can hardly be treated by the aforementioned chemical approach. For this, the physics of complex systems can be an alternative way deserving attention [10]

(2) To make the concept of amphiphile more actual. Usually, amphiphiles are modelled as simultaneously having a polar and an apolar part within their molecular architecture. These two parts have been always intended as moieties. So, traditionally, an amphiphile has one polar moiety and one apolar one. Here, we want to use a more useful and general concept of amphiphile, which can eventually have one or more polar and one or more apolar part functional groups. With the intervention of organic chemistry synthesis, it is common to deal with complex molecules with a lot of functional groups with different polarities, so the latter definition of amphiphile can be more satisfactory in many contexts. The presence of many different functional groups of course enriches the scenario of possible interactions and consequently the overall self-assembly, thus rendering the above cited vision based on the physics of many-body complex systems more adequate

These new concepts are schematically depicted in Figure 1.

In this ambit, small amphiphiles are versatile molecules since they have a reduced steric hindrance allowing a higher orientational freedom in their self-assembly thanks to their reduced size. In addition, if various polar and various apolar groups are concurrently present in different locations of the molecule, then a complex distribution of polar and apolar interactions is foreseen giving enriched scenario of selfassembly possibilities. At the same time, noble metals have unique photophysical and chemical properties. When the building blocks are amphiphiles based on luminescent metallorganic or coordination complexes having sensitive photophysical properties, advanced dynamic functional systems are obtained with striking possibilities of applications in major fields like biomedical, electrooptics, sensing, etc. [1116]. In this tutorial, we review the basic concepts on selfassembly of small amphiphilic molecules with metals that give supramolecular systems with luminescent properties. Besides the applications envisaged, these systems may bring important advances in fundamental research, by understanding the mechanism of self-assembling through weak intermolecular interactions which is ubiquitous in nature, thus permitting a better understanding of the processes in the living matter [17]. Importantly, the modification of the photophysical properties as a function of molecular environment can be used as a tool to investigate the molecular environment or the assembling mechanism.

While there is plenty of works focusing on the aspects of self-assembly, preparations of metal-amphiphile assemblies, and their applications in photophysics, the novel aspect of this contribution is to furnish to the reader a panoramic view of this exciting problematic in the fascinating framework/formalism of physics of complexity, simultaneously offering a concrete and updated picture of the state-of-the-art of the published works.

We wanted to prepare an easy-to-read critical summary interconnecting different disciplines like physics of complex systems (i.e., those systems where the overall properties cannot be described in terms of the properties of their constituents since emerging properties arise due to peculiar interactions, synergistic effects, and collective phenomena), coordination chemistry, and applications, with the final aim to tickle the reader's imagination and to hopefully stimulate new ideas and research.

\section{Amphiphiles and Their (Self-)Assembly with Metals}

The term amphiphile is quite general, since it refers to any kind of molecule simultaneously possessing both a polar and an apolar part within its molecular architecture. Even the presence of a very small polar group, like $-\mathrm{OH}$, in a much wider apolar structure, like in cholesterol, can confer 

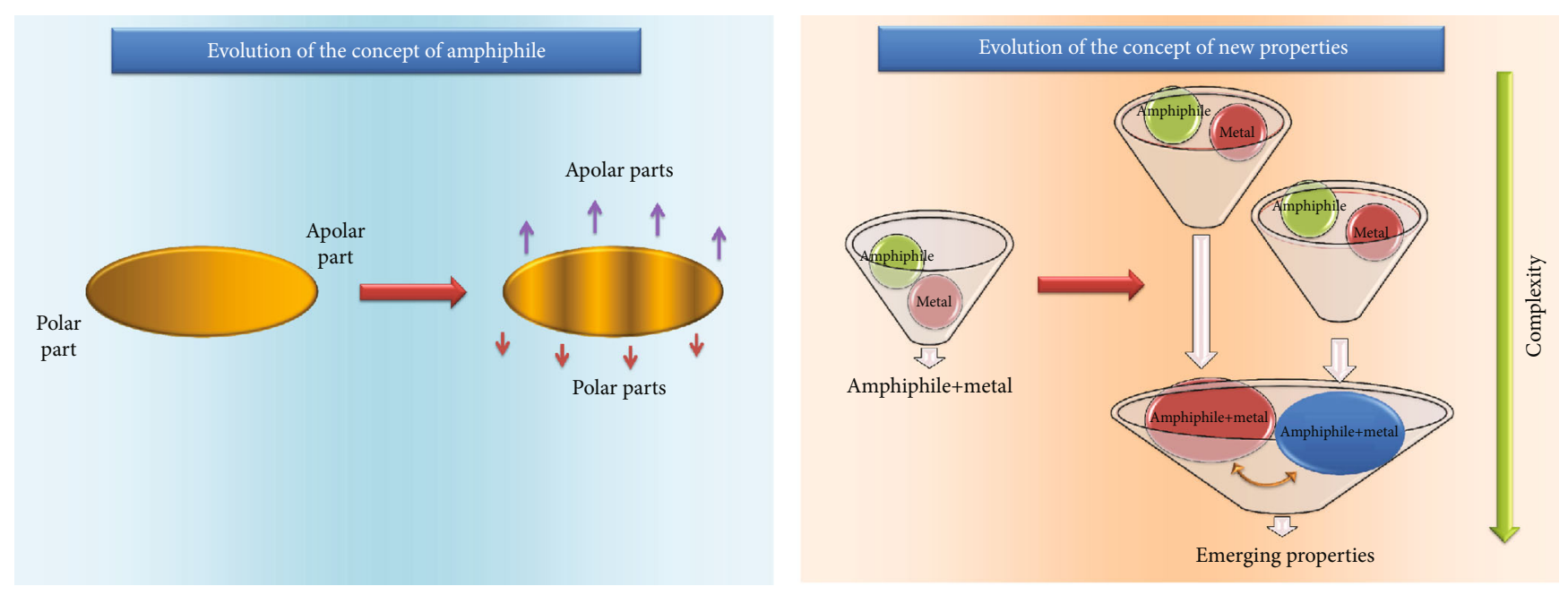

FIGURE 1: Schematically representation of new amphiphile concepts.

amphiphilic properties. Indeed, cholesterol tunes membrane fluidity within the phospholipidic bilayer of cell membranes due to its amphiphilic properties.

Although there are many works covering all the specific aspects involved in the self-assembly of such molecules, a critical and multidisciplinary treatment, giving sight from the top of all the aspects, can be of benefit for researches approaching this topic. At the same time, specific references will be also shown as sources of further details.

It is obvious that amphiphilicity triggers the simultaneous presence of different types of intermolecular (noncovalent) interactions: $\mathrm{H}$-bonds, van der Waals interactions, $\pi-\pi$ ones, electrostatic effects, and so on. A detailed description of such interactions and their entities is reported in Reference [5]. If such interactions are weak (so called "soft interactions" of the order of few $\mathrm{kJ} \cdot \mathrm{mol}^{-1}$ ) but a multiplicity of interaction sites is present, then several mechanisms of structuring are possible [18-20] giving the field of soft-matter science: polymeric materials, membranes, quaternary structures of proteins, colloids, foams, detergents, and ionic liquids [21-23] with an overall effect strong enough to hold together different building blocks sometimes having different molecular structures [24-26].

However, even the presence of a reduced number of weak interactions of the order of the $K_{B} T$ factor (i.e., the typical thermal energy at temperature $T$, where $K_{B}$ is the Boltzmann constant) at room temperature can give reversibility which is one key characteristic of stimuli-responsive smart materials.

In this ambit, comprehension and detailed treatment of the main forces acting in nanostructures (hydrogen bonding, hydrophobic effects, screened electrostatic interaction, steric repulsion, and van der Waals forces) are necessary [27-29].

The metal can be bonded by opportune polar functional groups of the amphiphiles which are able to form coordination bonds (like hydroxyl, amino, carboxylic, etc. groups). However, there are two basic phenomena at the bottom of the building up of amphiphile-metal assemblies:

(1) The inclusion of metal-containing species (salts, coordination complexes, nanoparticles, etc.) within the compartmentalizing domain formed by the selfassembly of amphiphiles

(2) The direct interaction between the metal atoms and the amphiphilic molecules

Let us see these two contributions separately.

2.1. Self-Assembly of Amphiphiles and the Inclusion of Metal Atoms in Convenient Domains. Here, it must be preliminarily shown the distribution of polar and apolar sites in amphiphile-based complex systems. The morphologies of the assembled structures can be various and depend on several parameters: composition, surfactant concentration, and type of amphiphiles as well as temperature and pressure. Figure 2 shows some examples of possible structures.

Such structures can be found in two-component (amphiphile/solvent system) but also in three-component systems (polar/amphiphile/apolar) whose aggregation pattern can be described by triangular phase diagrams.

Generally speaking, the surfactant concentration is of utmost importance: at very low concentration, the entropic driving force makes molecules randomly dispersed, whereas at higher amphiphile concentration, their interactions gives an enthalpic contribution and make them assemble. Anisotropy of structures, bicontinuity of microphases, and liquid crystals are always the consequence of the balance of intermolecular interactions and steric interactions [30-32].

Nonionic amphiphiles [33] have even more complex aggregation patterns. For them, usually a cloud point exists, as a consequence of collective phenomena involving a large number of macromolecules [34]. Temperature effects, of course, cannot be neglected in this situation.

In this scenario, there are different possibilities to find sites for metal binding, which usually are bound to the polar groups. So, the domains formed as a consequence of the polar/apolar nanosegregation can act as local, compartmentalizing nanoreactors, in few words exerting templating functions. 


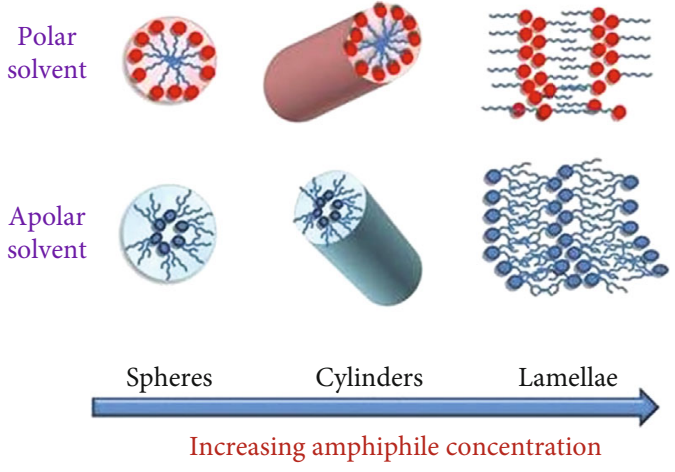

FIgURE 2: Some structures formed by the aggregation of amphiphiles. They can be direct (upper) or reversed (lower) depending on the polarity of the solvent.

However, they must not be considered as rigid parts nor closed substructures: the weakness of the interactions involved makes them evanescent and characterized by a wide variety of dynamical processes (e.g., conformational change of monomers, lateral diffusion of monomers within the aggregate, and aggregate breaking/reforming/scission/shape fluctuation), each one with a characteristic time scale. These aspects are clearly discussed and commented on in an easyto-read minireview by Calandra et al. [10]. Therefore, such structures must be considered only in terms of time- and space-averaged systems.

The shape of such aggregated structures can be controlled considering the so-called critical packing factor (Cpp) introduced by Israelachvili $[35,36]$ and defined as

$$
\mathrm{Cpp}=\frac{V_{0}}{A_{\mathrm{mic}} l_{c}},
$$

where $V_{0}$ is the volume of the hydrophobic chains, $l_{c}$ is the maximum effective length (critical chain length), and $A_{\text {mic }}$ is the effective hydrophilic head group surface area.

Changing molecular shape gives different $V_{0}, l_{c}$, and $A_{\text {mic }}$ and, consequently, different Cpp values which, synthetically, are correlated by different structures, as summarized in Table 1.

This gives the researcher the opportunity to prepare, by organic synthesis methods, ad hoc molecules having desired morphologies; synthetic or natural phospholipids which are generally made up of one hydrophilic head and two hydrophobic tails $[37,38]$, for example, give usually closed amphiphile bilayer structures (liposomes). In micelles, the radius is usually $5-50 \mathrm{~nm}$ [39] while in vesicles, the size usually spans around $10 \mathrm{~nm}-10 \mu \mathrm{m}$ due to concentric bilayer surfaces in an onion-like structure [40-42]. More complex structures of amphiphiles are of course more difficult to rationalize due to complex synergistic effects $[43,44]$.

The inclusion of metal-based compounds may influence the phase diagram of the surfactants in water in either cooperative or destructive ways, depending on their nature. For example, transition metal complexes with an overall hydrophobic nature will be hosted in the hydro-
TABLE 1: Typical structures obtained by the various critical packing factors (Cpp).

\begin{tabular}{lc}
\hline Cpp & Structure \\
\hline$<1 / 3$ & Spherical \\
$1 / 3-1 / 2$ & Cylindrical \\
$1 / 2-1$ & Vesicles (spherical or ellipsoidal) \\
1 & Lamellar \\
$>1$ & Inverse micelles \\
\hline
\end{tabular}

phobic core of the micelles, while water soluble complexes will be part of the corona shell or solvated in the water media. Accurate photophysical investigations in the case of luminescent complexes may represent a key tool to investigate the exact location of the complexes $[45,46]$.

2.2. Amphiphile-Metal Interaction. Direct amphiphile polar head-metal atom interaction can be quite strong, thus giving a marked contribution to the overall self-assembly. Usually, Lewis bases donate electron density to the metal centre which behaves as Lewis acid. According to the covalent bond classification proposed by Green and Parkin [47], there are different types of ligands. These are

(1) L-type ligands (neutral two-electron donors like in metal-ligand with dative interaction)

(2) X-type ligands (where one electron comes from the metal and the other from the ligand)

(3) Z-type ligands (if the metal is an electron density donor towards the ligand)

It is interesting to note that it can be regarded as a categorization based on the type of interactions rather than being a classification of the type of ligands. The type and strength of such interactions can depend on several factors: electrostatics and orbital overlap [48] can give an estimation of the metalligand bond strength.

Complexes of transition metals form strong bonds with hard Lewis basic ligands like those having $\mathrm{N}$ - and $\mathrm{O}$ - donor atoms [49]. Some estimation of bond strengths [50] are reported in Table 2 and compared with $\mathrm{H}$-bonds and typical C-C single bonds.

This clearly indicates that the metal-ligand bond is quite strong. It is so strong that it can be considered as a driving force in soft matter self-assembly. The strength of a bond, indeed, is directly related to the energy that the system gains during its formation: the stronger the bond, the higher the amount of energy involved and, consequently, the stronger the tendency of the system to follow that specific reaction/change pathway. We can also suggest the following relation between bond valences $(v)$ and bond lengths even if correlation between bond strength and bond lengths is still under discussion [51]: 
TABLE 2: Energies of some representative bonds.

\begin{tabular}{lc}
\hline Bond & Energy $(\mathrm{kJ} / \mathrm{mol})$ \\
\hline $\mathrm{Cu}-\mathrm{N}$ bond in $\left[\mathrm{CuCl}_{2}\left(\mathrm{NH}_{3}\right)_{2}\right]$ & 90 \\
$\mathrm{Zn}-\mathrm{O}$ in $\left[\mathrm{Zn}_{4} \mathrm{O}\left(\mathrm{O}_{2} \mathrm{CC}_{6} \mathrm{H}_{4} \mathrm{CO}_{2}\right)_{3}\right]$ & 180 \\
$\mathrm{H}-$ bond & $10-100$ \\
$\mathrm{C}-\mathrm{C}$ covalent bond & 350 \\
\hline
\end{tabular}

where $R$ is the experimental single bond length, $d$ is the bond distance, and $b$ is the Brown-Altermatt constant $(b=0.37 \AA)$. A bond length of $>3.5 \AA$ corresponds to a valence close to zero. This is just an example showing the usefulness of the valence bond theory. This theory, focusing on how the atomic orbitals combine to give individual chemical bonds when a molecule is formed, is simple and of practical use in the case of metal-molecule self-assembly, where the high number of electrons typically possessed by the metal can sometimes present problems. Furthermore, in a modern vision of the valence bond theory, the overlapping atomic orbitals are replaced by the overlapping of orbitals expanded over a large number of basic functions, which gives a competitive way to calculate reliable energies in quantum mechanics procedures [52], where again, the high number of metal electrons generally constitutes a difficulty to overcome. However, for details on valence bond theory, the curious reader is referred to Reference [53].

The bonds with metallic species can be exploited for surface stabilization/functionalization in nanoparticle capping (see later), and their further ordering can give the so-called "metamaterials" with emerging/collective magnetic, optical, and electronic behaviours, of precious use in nanoelectronic and nanophotonic $[54,55]$. In this ambit, the organicinorganic interface is of utmost importance [56] and is exploited in magnetic nanoparticles for bioapplications, core-shell structures for simultaneous magnetic resonance (MR), and fluorescence imaging and for drug delivery [57, 58]. For a panoramic view of all these aspects, see the review by Wei et al. [59].

Having explored the aspects involved in amphiphilemetal organization, we now start showing the state-of-theart of the amphiphile-noble metal complexes where the photophysical properties of the self-assembled structures are investigated.

\section{Supramolecular Assembly and the Final Structure}

Once we have shed light on the basic principles for amphiphile-metal bonding and assembly, we must now face the problem of how such assembly interacts to form the final structure. The idea of self-assembled structures of amphiphiles working as templates for metal hosting must somehow be completed since amphiphile self-assembly can be, in turn, dependent on the presence of the metal and vice versa. On the other hand, the amphiphile-metal interactions become in competition with the huge number of other interactions which, even if of lower intensity, can be prominent due to their high number of sites. This situation is typical in soft matter [60].

The hydrophobicity/hydrophilicity balance turns out to be not the unique driving force, since $\pi-\pi$ interactions, van der Waals interactions, H-bonds, and even metal-metal, metal-ligand, ligand-ligand interactions are also present [61], enriching a scenario typical of complex systems. In this framework, different kinds of assembly can be present:

(i) Isodesmic assembly, where the change in the free energy for the addition or subtraction of a molecule from an assembly is independent of the size of the assembly

(ii) Cooperative assembly, where more than one molecule is involved in creating or destroying an assembly, so a specific number of molecules are necessary to form an assembly. In such a process, interactions between the molecules play a necessary role

(iii) More complicated processes of assembly (quasi-isodesmic assembly, activation and growth assembly, nucleation and growth assembly, etc.), behaving as a combination of simple processes

For a review of such mechanisms, see Reference [62].

The mechanism of aggregation is hard to foresee due to the complexity of the systems. Generally speaking, upon gradual increase of hydrophilicity of the amphiphile backbone, a change from cooperative to isodesmic is observed. It is believed that cooperative self-assemblies occur only in specific hydrophobic/hydrophilic patterns in a delicate balance of all the other interactions like metal-metal, $\pi-\pi$ interactions, and $\mathrm{H}$-bonds which synergistically assist the building up of the self-assembled structures. The more complex and/or cumulative driving forces with respect to the classical low molecular weight surfactants may be one reason for the lowering of the critical aggregation concentration (CAC) with one or even two orders of magnitude, observed in some supramolecular assemblies based on metal complexes.

In Figure 3, the delicate equilibrium among all these interactions in sustaining the self-assembled structure is schematically depicted.

This can be taken as a first hint that amphiphile selfassembly and the direct amphiphile-metal interactions are, as a matter of fact, interconnected. In the following paragraph, we will show some applications in support of this view.

3.1. Some Applications: Evidences That Amphiphile SelfAssembly and the Direct Amphiphile-Metal Interactions Are Interconnected. The self-assembled structures of amphiphiles can also host molecule clusters [63], thus allowing their stabilization. Metal nanoparticles are also usually synthesized within the polar core of reversed micelles. A precursor salt, being polar, is solubilized in the confined space of the polar domains and then reduced by addition of a convenient chemical species [64]. Also, semiconductors (CdSe, ZnS, CdS, etc.) are synthesized exploiting the segregation properties of polar domains. See, for example, the review by Eastoe et al. and 


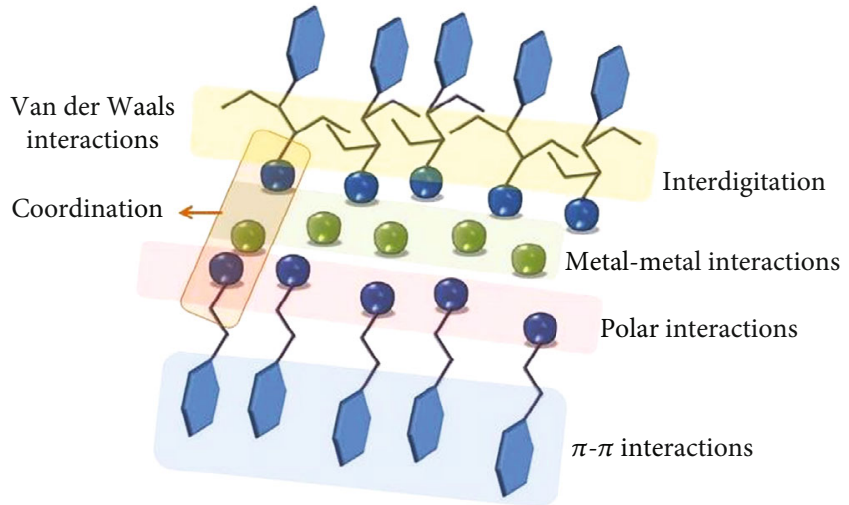

FIGURE 3: Scheme of the interactions involved in amphiphile-metal self-assembled structures.

references therein for a more complete view [65]. In all these cases, a direct interaction of the amphiphile polar head and the particles hosted is claimed.

Self-assembled nanostructures can also present interesting morphological [42] characteristics and dynamic behaviour [66]. They can be exploited for the synthesis of zeolites [67, 68], the formation of peptides and proteins for nanotechnological applications [69] including the preparation of protein fibres [70], conductive fluids [71, 72], and inorganic metal-semiconductor nanoparticles with novel optical properties [73]. In this ambit, the presence of metal and its interaction with amphiphiles open new scenarios in the design and fabrication of novel materials [74].

The bonds between the surfactants and metal ions constitute interactions used in water purification field. Solvent extraction processes can be used for separation of rare earth elements in hydrometallurgy [75].

Usually, derivatives of phosphoric acid are used as follows: besides the commonly used bis(4-ethylcyclohexyl) phosphoric acid [76], also bis(2-ethylhexyl) phosphoric acid (HDEHP) finds applications as, for example, in cobalt and in lanthanide separation [77, 78]. It is interesting to notice that although HDEHP has been defined "an excellent extractant of rare earth metal ions" in the article by Yuan et al. [79], in the same article, the authors mixed it with a cationic trimethyltetradecylammonium hydroxide $(\mathrm{TTAOH})$ in water, generating a birefringent LR phase consisting of densely stacked multilamellar vesicles. As can be seen, the two aspects that we have presented distinctly for didactical purposes are, as a matter of fact, interconnected. Indeed, the alkyl phosphate tendency to bind metals can cause the formation of a layer of opportunely oriented molecules onto metal surfaces for its protection: Guo et al. [80] investigated the formation of triphenyl phosphate and bis-(2-ethylhexyl) phosphate films on iron surface using both experimental methods and molecular simulations. This phenomenon inspired their use as protective agent (inhibitors) towards tarnishing in silver [81].

Luminescent micelles are attractive for imaging biological tissues; thus, in recent decades, efforts have been devoted for their obtainment, mainly by the encapsulation of emitters within the micelle cores resulting in luminescent micellar coassemblies [82-84]. Ultrasmall nanomicelles formed in water by semiconducting polymer dots containing phosphorescent $\operatorname{Ir}(\mathrm{III})$ complexes were showed to pass across the cytoplasm cell membrane and by irradiation generate singlet oxygen that induces effectively the apoptosis and death of tumor cells [85]. Similarly, dual-emissive conjugated polymer dots in phosphate buffer solution containing a phosphorescent $\mathrm{Pt}(\mathrm{II})$ porphyrin complex were shown to exhibit excellent intracellular ratiometric oxygen sensing properties in living cells, and the luminescence imaging of a tumor hypoxia in mice was performed by oxygen sensing experiments [86]. $\mathrm{Pt}(\mathrm{II})$ complex encapsulated in the hydrophobic core of some poly( $\varepsilon$-caprolactone)s were investigated as extracellular or intracellular multiparameter sensing systems [87].

Metal-based surfactants may directly form micellar structures in water, with unique supramolecular structures and sensitive properties easily tunable by external agents. The advantages over their organic counterparts are resulting from their enhanced photostability, long luminescent lifetimes that remove background interferences, and large Stokes shifts [88]. For example, a water soluble tris-cyclometalated Ir(III) complex having polar side chains based on oligo(ethylene glycol) with a terminal ammonium alkyl group, forming nanoparticles in water, was shown to be effective as a cell imaging agent due to proper cellular uptake, high emission brightness, and nonlinear absorption ability at submicromolar concentration [89].

Finally, we would like to point out that there can be interesting uses also in catalysis [90].

The peculiar self-assembly of amphiphiles and their tendency to bind metal surely make these substances an interesting class of materials to be tailored for specific applications in catalysis.

\section{Supramolecular Aggregates of Luminescent Transition $d$-Block Metal Complexes in Water}

Several strategies have been adopted in order to induce supramolecular assembling properties in water for $d$-block metal complexes, principally due to the facility of obtaining heteroleptic species, with two or even three different nature ligands. Given the intrinsic hydrophobicity of a metal complex formed upon coordination with two or three chelating ligands, the simplest way to induce amphiphilicity is to graft hydrophilic chains on one of the ligands (Figure 4), of polyethylene glycol (pEG) type, yielding surfactant type complexes. The major difference from the classical surfactants, however, is the change of the nature of the hydrophilic/hydrophobic part, respectively in the case of classical surfactants the hydrophobic part is formed by fluid alkyl chains. Other strategies are based on grafting neutral or charged solubilizing groups on one of the ligand (Figure 4, B), conferring thus hydrophilicity to the metal complex, while the hydrophobicity is ensured by the grafting of alkyl chains on a coligand. Not lastly, introduction of solubilizing counterions in charged complexes may 


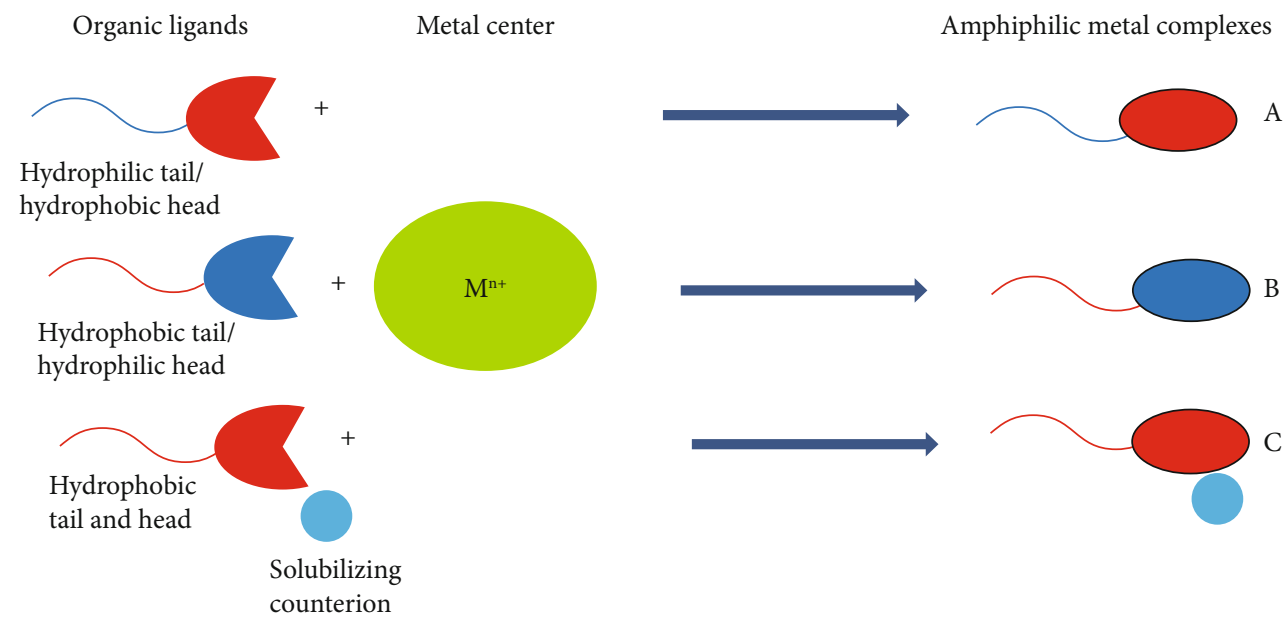

FIGURE 4: Schematic illustration of different types of amphiphilic complexes and the resulting hydrophilicity/hydrophobicity nature after functionalization.

confer the required amphiphilicity for self-assembly (Figure 4, C). Therefore, it would be highly interesting to review the advances obtained up to date in this field, in an attempt to explore the factors that link the molecular structures with the self-assembling abilities and the shape and morphology of the supramolecular dynamic structures obtained in aqueous media. Our attention will be focused on supramolecular dynamic systems formed by luminescent coordination or metallorganic complexes, highlighting the changes of the photophysical properties as a function of the molecular environment, polarity, and medium rigidity.

$d$-block metals have a variety of oxidation states, coordination numbers, and geometries, which influence the shape of the resulting complexes and hence the ability of selfassembling into supramolecular structures. Indeed, it is relatively more easy to order flat structures, like square planar geometry complexes, than the voluminous tetrahedral or higher number coordination geometries. This may explain the increased number of square-planar Pt(II) amphiphilic complexes reported up-to-date with respect to other metal complexes that will be presented separately.

\subsection{From Luminescent Isolated Molecule to Supramolecular} Emitting Aggregates. Transition metal complexes based on $\mathrm{d}^{6}$ and $\mathrm{d}^{8}$ metal centres may exhibit emission from both ligand and metal centres, having excellent photostabilities, large Stokes shifts, and excellent emission efficiencies due to the high spin-orbit coupling constants induced by the presence of the heavy metal that permits populating the triplet excited states. Indeed, emission may occur from ligand transitions like intraligand (IL) or ligand-to-ligand charge transitions (LLCT), or metal transitions that involve metalto-ligand charge transfer (MLCT) or ligand-to-metal charge transfer (LMCT) electronic transitions. Moreover, many complexes may have triplet-state phosphorescence also due to metal-metal interactions or ligand-ligand stacking, like metal-to-ligand-ligand charge transfer (MLLCT), metalmetal-to-ligand charge transfer (MMLCT), or ligand-tometal-metal charge transfer (LMMCT).
Finally, the complexes have long-lived emission lifetimes due to the spin-forbidden nature of the relaxation from the triplet state to the ground state. The photophysical properties of transition metal complexes are considered lately to be a winning strategy for applications in biomedicine as therapeutic and/or diagnostic agents [10, 11, 91-95].

However, in the aggregate state, new excited states may be formed, different from those exhibited by the isolated molecule, yielding different decay pathways that may be (i) nonradiative-this phenomenon being defined as aggregation-caused quenching (ACQ) or radiative, the socalled aggregation-induced emission (AIE). These new excited states may be formed in the ground state or in the excited states, respectively, through excimer formation. Moreover, the aggregation may induce AIE by restricting the intramolecular motions (RIM), by steric hindrance or by hindering the access of oxygen, a well-known quencher of phosphorescent probes. Finally, the photophysical properties of transition metals are sensitive to the molecular environment; therefore, they can be used not only for sensing but also for proving the supramolecular assembling in dynamic systems like the examples presented further (vide infra).

\subsection{Surfactant-Type Transition Metal Complexes}

4.2.1. Pt(II) Amphiphilic Coordination Complexes. Several research groups obtained important results with $\mathrm{Pt}$ (II) complexes, exploiting the flat coordination geometry of the square planar metal centre that makes them more prone to be piled up into supramolecular assemblies. In certain conditions, Pt...Pt metallophilic interactions may be formed wherein one side contributes to the self-assembling, while on the other side, the close vicinity of the metallic centres induces the formation of new orbitals due to $\mathrm{d}_{\mathrm{z}}{ }^{2}$ interactions leading to metal-metal-to-ligand charge transfer (MMLCT) states. Thus, luminescence switching between different emitting states due to the arrangement into supramolecular "soft" dynamic sheet-like, micellar, vesicular, rod-like, or fibrous structures triggered by external stimuli like concentration, 
Polar solvent: reverse mode

Hydrophobic head

Hydrophilic tail

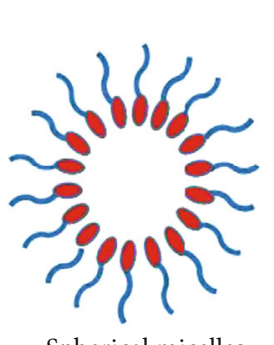

Spherical micelles
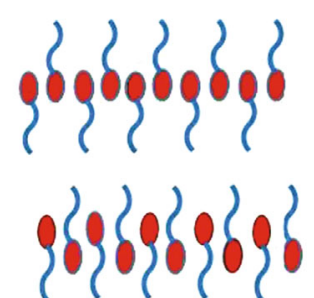

Layer arrangements: with and without metal-metal interactions

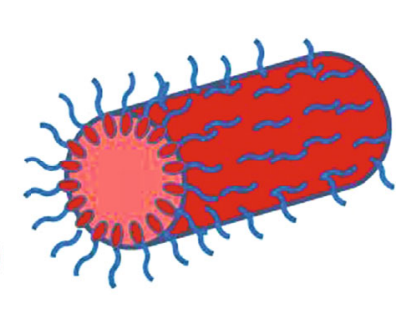

Fibres

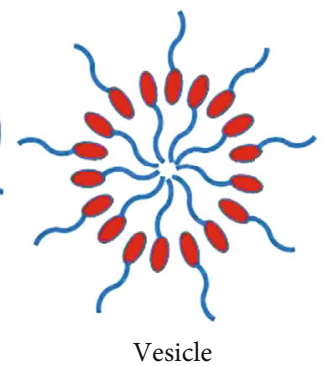

Vesicle

Hydrophilic head

Polar solvent: classical mode

Hydrophobic tail

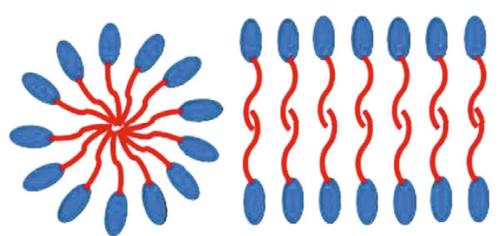

Spherical micelles

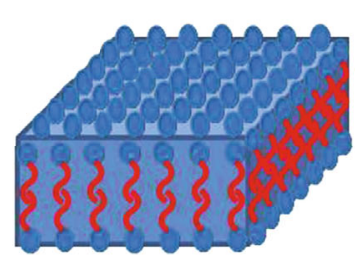

Bilayer sheet

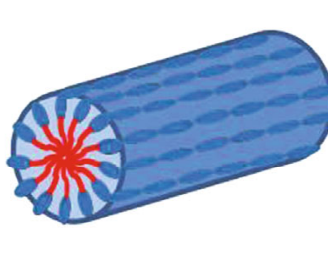

Rod-like micelles/fibres

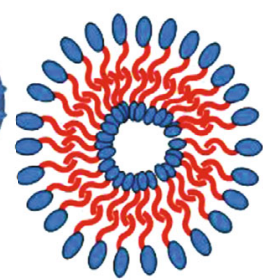

Vesicle

FIGURE 5: Schematic representation of the possible supramolecular assemblies of amphiphilic coordination complexes in aqueous media.

temperature, and presence of nonsolvent may be obtained. As a function of substituents and, respectively, the hydrophobic/hydrophilic unit nature, the possible structures formed by amphiphilic surfactant-type Pt(II) coordination complexes in water are represented in Figure 5.

The stronger the Pt...Pt interaction (shorter distances), the more bathochromically shifted is the MMLCT band, and this property can be used to investigate the formation of supramolecular soft nanostructures in solution, as demonstrated by several groups whose research is presented as follows. The Pt(II) complexes are divided as a function of the ligand type.

(1)Pt(II) Complexes Based on Bis(triazolyl)pyridine-Type (tzpy) Ligand. Aliprandi et al. reported the synthesis of an amphiphilic neutral $\mathrm{Pt}(\mathrm{II})$ complex with a tridentate $\mathrm{N}^{\wedge} \mathrm{N}^{\wedge} \mathrm{N}$ ligand based on a functionalized bis(triazol-5yl)pyridine (tzpy) and an $\mathrm{N}$-donor pyridinic coligand substituted with a triethylene glycol (3EG) chain (complex Pt_1 in Figure 6) [96]. The smart design provided a hydrophobic flat chelate unit having a hydrophilic pendant chain and an $\mathrm{NH}$ function on the coligand able to participate to the assembly through directional H-bonds. In a dioxane solution, the complex exists in molecular solvated form, displaying a weak blue luminescence $(\Phi=0.01)$ and a short excited lifetime $(\tau=2.6 \mathrm{~ns})$ arising mainly from a triplet-ligandcentred $\left({ }^{3} \mathrm{LC}\right)$ state. The complex belong to a family of neutral $\mathrm{Pt}(\mathrm{II})$ complexes able to self-assemble into highly emissive nanosheets, nanowires, or polymeric supramolecular structures essentially through metallophilic, hydrophobic, and $\pi-\pi$ stacking interactions $[97,98]$.

By playing with solvent composition and light, three different supramolecular assemblies were successfully entrapped and characterized as two kinetically metastable and a thermodynamically stable form. Flash injection of a dioxane solution of Pt_1 into water yielded a metastable kinetic state in which the molecules form soft vesicles with a hydrodynamic diameter $\left(D_{h}\right)=126 \mathrm{~nm}$, having the $\mathrm{Pt}(\mathrm{II})$ complexes in the inner core with short Pt $\cdots$ Pt metallophilic interactions and the hydrophilic 3EG tails facing the polar media (reverse mode vesicle, Figure 5). The aggregates showed a strong emission in the orange region of the visible spectra with a long excited state lifetime value $(\Phi=0.84, \tau$ $=646 \mathrm{~ns}$ ). The system interconverts in time into a thermodynamically stable isoform, where the complexes are arranged into micrometer-long fibre structures with optical properties similar to those of the monomeric specie, indicating the absence of the Pt...Pt interactions (Figure 5-layer arrangement without metal-metal interactions). However, the 
<smiles>[R]c1ccncc1</smiles>

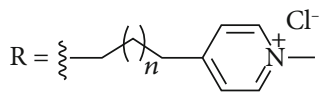

Pt_3a: $n=0$

Pt_3b: $n=3$

Pt_3c: $n=6$

Pt_3d: $n=9$

Pt_3e: $n=12$

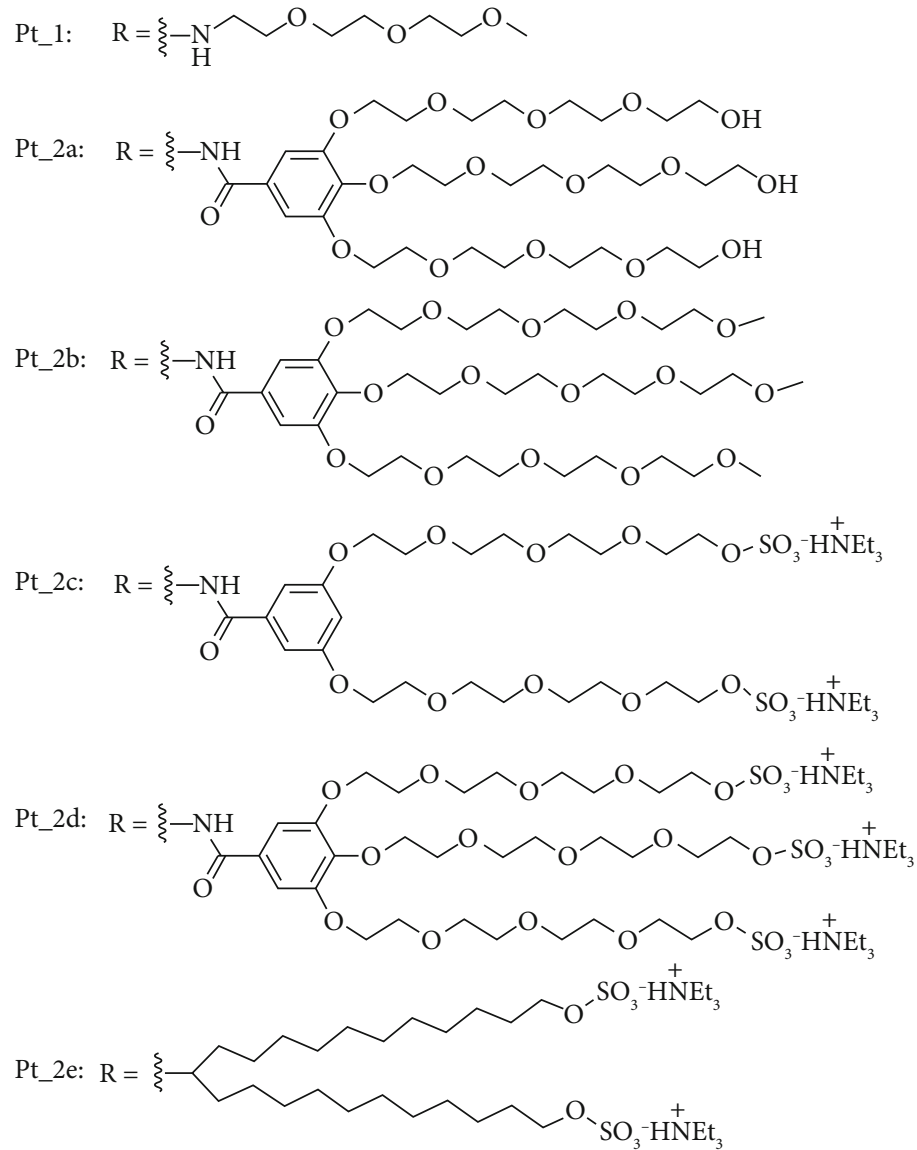

FIGURE 6: Chemical structure of the family of Pt(II) complexes based on (2,6-bis(3-(trifluoromethyl)-1H-1,2,4-triazol-5-yl)pyridine) as tridentate ligand.

emission quantum yield and lifetime values of the system are increased with respect to the single molecule, due to the enhancement of rigidity and shielding of the oxygen quenching $(\Phi=0.20, \tau=1080 \mathrm{~ns})$. UV-vis experiments under thermodynamic control showed that the mechanism of assembly of the fibre structures is a cooperative nucleationelongation process, while for the nanoparticles, an isodesmic assembly was revealed. During kinetic studies, a second metastable form was unrevealed, which was successfully entrapped by photochemical conversion through irradiation of the thermally stable fibre assembly. The second kinetic metastable structure is formed by green-emissive fibre assemblies in which the complexes are not isolated as in the thermodynamically stable fibre structure, being arranged in a face-to-face fashion like in the metastable vesicular assembly, however, having larger distances between the metal centres (Figure 5-layer arrangement with metal-metal interactions). Importantly, the different photophysical properties of the aggregates were used as a fingerprint to obtain a real-time visualization of the dynamic evolution of the assemblies.

Further, the modulation of the number, charge, and nature of the hydrophilic group was achieved by grafting on the pyridinic coligand through an amide linkage a gallate unit substituted with several hydrophilic flexible groups (complexes Pt_2a-e in Figure 6) [99]. For complexes Pt_2a$\mathrm{b}$, the hydrophilic unit is comprised of neutral tetraethylene glycol (4EG) chains, while for complexes Pt_2c-d, the hydrophilicity of the flexible $4 \mathrm{EG}$ chains is reinforced by negatively charged terminal sulfate units. Pt_2e has an extended hydrophobic part comprised of both a rigid complex cation and fluid undecyl alkyl chains that is balanced by small hydrophilic negative charged groups as terminal units. All complexes Pt_2a-e aggregate in water and have CAC in the range of $10-50 \mu \mathrm{M}$. The modulation of the substituents on the pyridinic coligand yielded the self-assembly into different morphology nanoparticles. Complexes Pt_2a-d form in aqueous TRIS buffer aggregates with the hydrodynamic diameter $\left(D_{h}\right)$ in the size range of $5-25 \mathrm{~nm}$, while complex Pt_2e, with a larger hydrophobic part, forms larger fibre particles with $D_{h}$ of $150 \mathrm{~nm}, 100-300 \mathrm{~nm}$ length, and $6 \mathrm{~nm}$ width. The aggregates showed red-shifted emission with respect to the isolated molecules in THF and strong enhancement of the luminescence quantum yield $(\Phi=0.14-0.60)$ accompanied by long excited lifetime values (up to $550 \mathrm{~nm}$ ). The red-shift of the emission maxima suggests the formation of Pt...Pt interactions inside the aggregates. Tailored morphology and functionality luminescent virus-like particles were 
obtained using these Pt(II) aggregates as templates with capsid proteins.

Further, the pyridine coligand was parasubstituted with an alkyl chain terminated with a charged pyridinium unit acting as the hydrophilic moiety, similar with complex Pt_ 2e (complexes Pt_3a-e in Figure 5) [100]. Orange-red emission was obtained in water diluted solutions for all complexes $\left(c=5 \cdot 10^{-5} \mathrm{M}\right)$, attributed again to triplet ${ }^{3}$ MMLCT transitions due to strong electronic coupling between closed platinum centres. The variation of the chain length does not change the emission energy of the aggregates; however, lowering of the luminescence quantum yield was observed for shorter chain derivatives, most probably due to the proximity of the pyridinium unit that can quench the platinum emission through an electron transfer process. At very low concentrations $\left(c=10^{-6} \mathrm{M}\right)$, the complexes form a linear supramolecular polymer (layer arrangement in Figure 5) driven by $\pi-\pi$ stacking and Pt...Pt interactions, and with increasing concentration due to hydrophobic interactions, spherical aggregates of the vesicular/micellar type are formed. Upon encapsulation in silica matrixes, similar emission energy profiles are observed, however, with increased luminescence quantum yields and longer excited state lifetime values.

It is worth mentioning other analogue amphiphilic neutral Pt(II) complexes based on 2,6-bis(tetrazol-5-yl) pyridine and EG functionalized pyridine coligand used to build up supramolecular polymeric $1 \mathrm{D}$ and $2 \mathrm{D}$ nanostructures; however, no investigations in aqueous solutions were performed [101, 102].

A structurally similar class of $\mathrm{Pt}(\mathrm{II})$ complexes based on 2,6-bis(1-propanesulfonate-1,2,3-triazol-4-yl)pyridine and alkynyl aromatic substituents was designed and characterized by $\mathrm{Li}$ et al. [103]. Herein, the tridentate ligand is functionalized with hydrophilic substituents forming thus the polar part, while the coligand is included in the hydrophobic part of the molecule (complexes Pt_4a-c in Figure 7). At low concentration, the complexes show structureless emission bands centred in the red region $(672-702 \mathrm{~nm})$ derived from ${ }^{3}$ MMLCT excited states. The increase of concentration induces a small red-shift of the emission maxima and enhancement of the emission quantum yield, indication of an aggregation in water media assisted by metal-metal and $\pi-\pi$ stacking interactions. SEM and TEM images showed that while complexes $\mathrm{Pt}$ $4 \mathrm{a}$ and $\mathrm{Pt} \_4 \mathrm{c}$ form rod-like assemblies, complex $\mathrm{Pt}$ 4 $\mathrm{b}$ forms nanofibres (Figure 5-classical mode). By addition of tetrahydrofuran, a solvation of the aggregates formed in water followed by a reaggregation into analogous shape aggregates but with reverse morphology was induced with increasing THF content: while in water, the hydrophobic part of molecules are aggregated through aromatic stacking interactions of the phenyl aromatic rings with the heterocycles more dispersed and the sulfonates pointing towards water; in tetrahydrofuran/water $(9: 1 v / v)$, the hydrophilic sulfonates are aggregated and the phenylethynyl units are pointing towards the solvent (rod/fibre structures in classical mode in water and reverse mode in predominant tetrahydrofuran media-Figure 5).

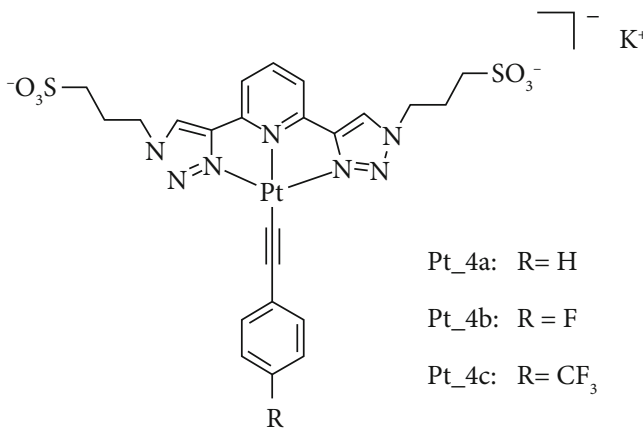

Figure 7: Chemical structure of amphiphilic Pt(II) complexes Pt 4a-c based on 2,6-bis(1-propanesulfonate-1,2,3-triazol-4yl)pyridine and alkynyl aromatic substituents.

(2)Pt(II) Complexes Based on Bis(benzimidazol)pyridineType (bzimpy) Ligand. Po et al. synthesized a series of amphiphilic Pt(II) complexes based on 2,6-bis(-benzimidazol-2' -yl)pyridine (bzimpy) and chloride or alkynyl ligands (complexes Pt_5a-c in Figure 8) [104]. The functionalization of the bzimpy ligand with sulfonate-pendant alkyl chains yielded an overall anionic nature of the complex, the charge being counterbalanced by $\mathrm{K}^{+}$cations. In water, the complexes exhibit weak luminescence at $\sim 675-683 \mathrm{~nm}$ ( $\Phi=0.032-0.111)$, the low-energy structureless bands being assigned as ${ }^{3}$ MMLCT emission due to formation of vesicular aggregates (classical mode-Figure 5) with diameters of about $200 \mathrm{~nm}$ by $\mathrm{Pt} \cdots \mathrm{Pt}, \pi-\pi$ stacking, and hydrophobic interactions. Interestingly, a second assembly process through a partial deaggregation-aggregation mechanism, yielding nanorods or nanofibres, was triggered by addition of increasing volumes of nonaqueous solvents like acetone, tetrahydrofuran, ethanol, or acetonitrile for complexes Pt_ $5 \mathrm{a}$ and $\mathrm{Pt} \_5 \mathrm{c}$. On the contrary, only a deaggregation process was observed for complex Pt_5b with addition of nonaqueous solvents. The absence of the second aggregation was explained by the presence of the bulky substituent trimethylsilyl (TMS) on the alkynyl hydrophobic coligand that likely prevented the reverse mode aggregation. The nanorods and nanofibres are built up by stronger Pt...Pt and $\pi-\pi$ stacking interactions than those in the vesicular aggregates, with the emission bands also of MMLCT nature shifted to slightly lower energies. The formation of vesicles or nanorods/nanofibres as a function of the solvent nature and polarity was explained by the different polar/apolar affinities exerted by the anionic sulfonate groups with respect to the $\operatorname{Pt}($ bzimpy) and the alkynyl coligand. The aggregation-deaggregationaggregation process is accompanied by solvatochromism, from red (vesicles) to yellow (small aggregates) and blue (complex Pt_5a) or magenta (complex Pt_5c) for the nanorods and nanofibres.

The introduction of an aromatic unit into the alkynyl coligand changes the shape of the supramolecular aggregates in water into $2 \mathrm{D}$ structures, dictated by the presence and length of alkyl chains grafted [105]. The complexes without long alkyl chains (complexes Pt_6a-c in Figure 8) form 


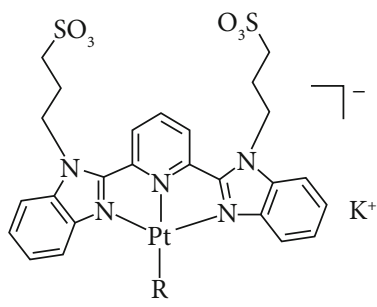

Pt_5a: $\mathrm{R}=\xi-\mathrm{Cl}$

Pt_5b: $\mathrm{R}=\xi-\mathrm{C} \equiv \mathrm{C}-\mathrm{C} \equiv \mathrm{C}-\mathrm{TMS}$

Pt_5c: $\mathrm{R}=\xi-\mathrm{C} \equiv \mathrm{C}-\mathrm{C} \equiv \mathrm{CH}$

Pt_6a: $\mathrm{R}=\xi \bar{\square}$

Pt_6b: $\mathrm{R}=\xi \equiv-\mathrm{CH}_{3}$

Pt_6c: $\mathrm{R}=\xi=-\mathrm{OCH}_{3}$

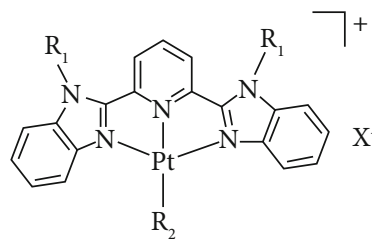

Pt_6d: $\mathrm{R}=\xi=\square-\mathrm{OC}_{7} \mathrm{H}_{15}$

Pt_6e: $\mathrm{R}=\xi=-\mathrm{OC}_{12} \mathrm{H}_{25}$

Pt_6f: $\mathrm{R}=\xi=\square-\mathrm{OC}_{16} \mathrm{H}_{33}$

Pt_6g: $\mathrm{R}=\xi=-\mathrm{OC}_{22} \mathrm{H}_{45}$

Pt_7a: $\mathrm{R}_{1}=y_{2} \mathrm{O} \sim \mathrm{O} \sim \mathrm{R}_{2}=$

Pt_7b: $\mathrm{R}_{1}=\xi-\mathrm{OC}_{12} \mathrm{H}_{25}, \mathrm{R}_{2}=\xi=$

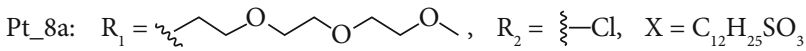

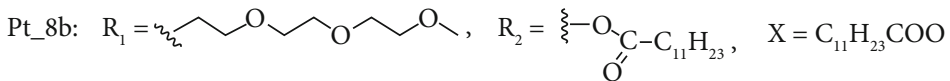

FIGURE 8: Chemical structures of the family of Pt(II) complexes based on 2,6-bis(-benzimidazol-2' -yl)pyridine as tridentate ligands.

sheet-like bilayered structures with the sulfonyl groups pointing towards the water and the core formed by $\operatorname{Pt}($ bzimpy) and the aromatic rings of the coligands (classical mode-Figure 5). With increasing chain length (complexes Pt_6d-g in Figure 5), nanofibres are formed, with diameters that increase gradually with the increase of the alkyl chain lengths (from 4 to $10 \mathrm{~nm}$ ) (classical mode-Figure 5). Both structures are stabilized by intermolecular Pt...Pt and $\pi-\pi$ stacking interactions having emissions originating from ${ }^{3}$ MMLCT excited states. The sheet-like nanostructures exhibit structureless emission bands centred at 693-698 nm, while the nanofibres exhibit more vibronic-structured emissions, with the maxima blue shifted towards lower energies. A further blue-shift is observed with increasing chain length (from $594 \mathrm{~nm}$ for complex Pt_6d to $644 \mathrm{~nm}$ for complex Pt_ $6 \mathrm{~g}$ ) indicating a closer packing of the emitters due to the compensation of the repulsive electrostatic interactions between the metal containing head groups by the increasing hydrophobic interactions.

The amphiphilicity of the $\mathrm{Pt}(\mathrm{II})$ complexes based on bzimpy and aromatic alkynyl was further modulated [106] by a consecutive substitution with two hydrophilic 3EG chains and two hydrophobic dodecyloxy alkyl chains in a reverse mode: complex $\mathrm{Pt}$ _7a has the hydrophilic chains grafted on the bzimpy ligand and the alkyl chains in the meta positions of the ancillary ligand, while complex Pt_ $7 \mathrm{~b}$ has the hydrophilic units on the ancillary ligand, the bzimpy being substituted with TEG chains (Figure 8). Surprisingly, only complex Pt_7a was soluble in water, forming nanostructures with morphology and photophysical properties depending on temperature, highlighting the delicate balance of intermolecular noncovalent forces needed for the assembly. At a low temperature in water $\left(5-27.5^{\circ} \mathrm{C}\right)$, complex Pt_7a forms sheet-like bilayered structures (few $\mu \mathrm{m}$ in size and $10 \mathrm{~nm}$ in height) (classical mode bilayered sheet-Figure 5), with a vibronic-structured ${ }^{3}$ IL band centred at $568 \mathrm{~nm}$, while with increasing temperature (range of transition temperature between 30 and $42.5^{\circ} \mathrm{C}$ on heating), micellar supramolecular structures (classical mode-Figure 5) are formed (diameter $=8-19 \mathrm{~nm}$ ) with structureless emission originating from ${ }^{3}$ MMLCT excited states and a red-shifted maxima at $710 \mathrm{~nm}$, highlighting the formation of Pt...Pt and $\pi-\pi$ stacking interactions. The solubility of complex $\mathrm{Pt}$.7a in water was attributed to the ability of self-assembling into micellar structures due to the large hydrophilic head group that points toward water and a more condensed hydrophobic unit occupying a smaller area giving, thus, the necessary curvature to assembly into 
spherical micelles, while complex Pt_7b does not fulfill these requirements, hence resulting in being water insoluble.

Supramolecular amphiphiles were obtained with Pt(II) complexes based on bzimpy functionalized with 3EG chains and connecting anionic surfactants through donor or electrostatic interactions, respectively sodium dodecyl sulfonate (SDS) and sodium dodecanoate (SD) (complexes Pt_8a-b in Figure 8) [107]. Complex Pt_8a with only one hydrophobic chain formed in water solution rod-like aggregates (classical mode-Figure 5) of several $\mu \mathrm{m}$ length and $159 \pm 25 \mathrm{~nm}$ width, with a strong red emission centred at $618 \mathrm{~nm}$ consistent with the ${ }^{3}$ MMLCT state. Complex Pt_8b with an increased hydrophobic part with respect to complex Pt_8a formed sheet-like aggregates (classical mode-Figure 5). The CACs for both complexes reduced with two orders of magnitude with respect to their corresponding anionic surfactant precursors.

In the following, the two $\mathrm{Pt}(\mathrm{II})($ bzimpy) were linked by an oligo(p-phenyleneethynylene) ligand yielding dinuclear ionic Pt(II) complexes [108]. The peripheral bzimpy ligands were functionalized with hydrophobic alkyl or alkyne chains of different lengths, while on the alkynyl backbone, hydrophilic 3EG chains were grafted (complexes Pt_9a-l in Figure 9).

In DMSO solution the complexes aggregate into nonemissive plate structures with hydrodynamic diameters of ca. $1000 \mathrm{~nm}$ and minor involvement of Pt...Pt interactions. The addition of water until $20 \%$ induces a change in the morphology of the supramolecular structures from plate to fibres with a reduced dimension to ca. $800 \mathrm{~nm}$. The process is accompanied by a color change from yellow to orange associated to the switching on of Pt..Pt and $\pi-\pi$ stacking interactions in more polar media that induces a red phosphorescence with emission maxima at $\sim 700 \mathrm{~nm}$ derived from the formed ${ }^{3}$ MMLCT excited states. Upon further addition of water, another change of the morphology into spherical aggregates with even lower dimensions (ca. $100 \mathrm{~nm}$ ) is obtained, with increasing strength of metal-metal and $\pi-\pi$ stacking interactions, as showed by the increase of the emission intensity and a further redshift of the emission maxima. The photophysical properties were found to be influenced mostly by steric factors, while the self-assembling mechanism depends greatly by the hydrophobic/hydrophilic ratio. However, upon increasing alkyl chain length, a change in the mechanism assembly was observed from isodesmic (complex Pt_9a) to cooperative (complexes Pt_9b-d) and again to isodesmic (complex Pt_ 9e). The same change in the self-assembling mechanism was observed for complexes Pt_9d-l, where a cooperative mechanism could be achieved only for the complexes with balanced hydrophilic/hydrophobic interactions, while isodesmic mechanism was found for the complexes with increased hydrophylicity or hydrophobicity, respectively.

(3)Pt(II) Complexes Based on Terpyridine-Type (tpy) Ligand. Another highly versatile planar tridentate $\mathrm{N}^{\wedge} \mathrm{N}^{\wedge} \mathrm{N}$ ligand intensively used in obtaining emissive $\mathrm{Pt}(\mathrm{II})$ complexes is terpyridine (tpy) [109-111]. Yu et al. employed this ligand to obtain ionic amphiphilic Pt(II) complexes with alkynyl coligands that have covalently attached solubilizing neutral or charged groups (complexes Pt_10a-d, Figure 10) [112]. They aggregate in water-ACN mixture and aqueous buffer solutions showing a weak emission in NIR region; however, no further information about the supramolecular morphology was reported.

Subsequently, a comprehensive study of the morphology and photophysical properties of the supramolecular assemblies formed in water by $\mathrm{Pt}$ (II) complexes by a successive functionalization of tpy or aromatic phenylalkynyl ligands with hydrophilic 3EG chains was undertaken [113]. The molecular engineering of the chemical structures targeted successive increase of the hydrophilic part by functionalization with one, two, or three 3EG chains of the aromatic alkynyl ligand and presence of solubilizing $\mathrm{Cl}^{-}$counterion (complexes Pt_11a-c in Figure 10) and, respectively, the increase of the hydrophobic part by grafting two hydrophobic chains with increasing alkyl chain length on tpy and metathesis with OTf counterion, while maintaining constant the phenylalkynyl ligand substituted with two 3EG chains in the meta position (complexes Pt_11d-g in Figure 10). The study highlighted the need of a judicious molecular design to obtain stable supramolecular nanostructures: regarding complexes Pt_11a-c, only the first two formed micrometer long fibres (diameter: $5 \mathrm{~nm}$ for Pt_11a and $8-10 \mathrm{~nm}$ for Pt 11b) (reverse mode-Figure 5) with NIR emissive properties in water (structureless band centred at ca. $785 \mathrm{~nm}$ ) upon aggregate formation by increasing concentration, due to stacking of complexes by metal-metal and $\pi-\pi$ interactions. Although similar energies of the ${ }^{3}$ MMLCT emission bands suggest a similar strength $\mathrm{Pt} \cdots \mathrm{Pt}$ interaction in all three complexes, the presence of three hydrophilic 3EG chains prevents the formation of defined aggregate species.

Regarding complexes Pt_11d-g the functionalization of the tpy ligand with hydrophobic units induces insolubility in water, which was exploited to construct supramolecular assemblies in $\mathrm{DMSO} / \mathrm{H}_{2} \mathrm{O}$ mixture with increasing water content. The hydrophobic/hydrophilic ratio is also in this case necessary to be judiciously balanced. Complex Pt_11g with the longest octyldecyloxy chains precipitated directly on addition of water to the solution of DMSO containing the complex, while complex Pt_11f formed initially small spherical structures that grew into rod-like aggregates but collapsed back to small aggregates by increasing water content. Complexes Pt_11d and Pt_11e show similar behaviour, forming by an isodesmic process twisted fibrous supramolecular aggregates upon water addition in the DMSO solutions containing the dissolved complexes. Although they did not show luminescence in DMSO solution, upon addition of water the appearance and increase of low-energy ${ }^{3} \mathrm{MMLCT}$ emission band at ca. 770 and $710 \mathrm{~nm}$ for Pt_11d and Pt_ $11 \mathrm{e}$, respectively, showed the formation of aggregates through Pt $\cdots \mathrm{Pt}$ and $\pi-\pi$ stacking interactions, confirmed also by the color change from yellow to orange. TEM investigations showed that only complex Pt_11e was able to form well-ordered twisted fibres, while the images recorded for complex Pt_11d in DMSO/ $\mathrm{H}_{2} \mathrm{O}(9: 1 \mathrm{v} / \mathrm{v})$ showed the coexistence of tiny spherical fragments and long fibres. 


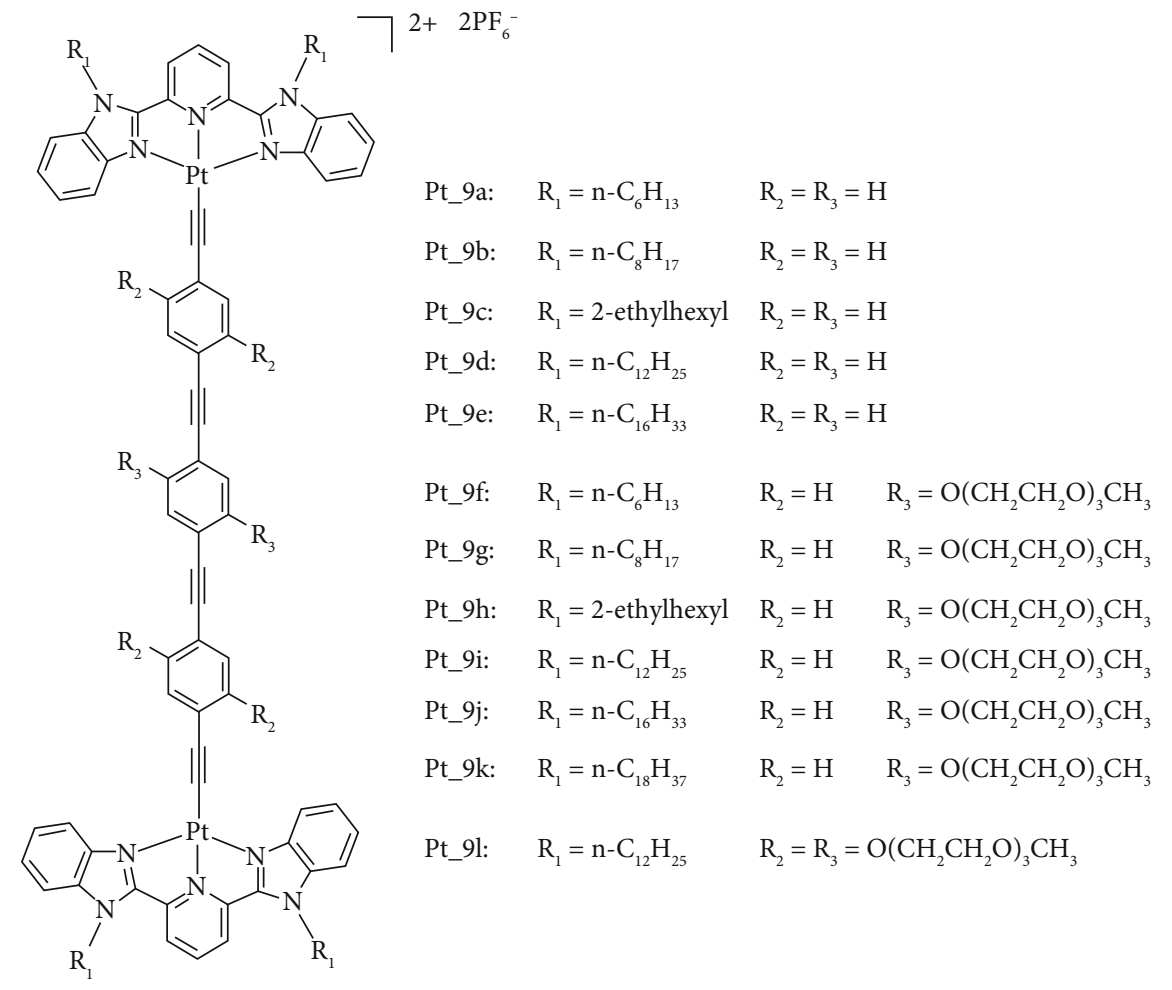

Figure 9: Chemical structures of dinuclear Pt(II) complexes Pt_9a-l.

(4)Supramolecular Pt(II) Amphiphilic Metallaclips. Supramolecular organoplatinum(II) metallaclips were obtained through a one-step coordination driven self-assembly by Wang et al. [114, 115]. Subsequent self-assembly into luminescent nanostructures built up by additional noncovalent interactions like hydrophobic, Pt $\cdots$ Pt and $\pi-\pi$ stacking took place in water. The design of the amphiphilic supramolecular coordination complexes was based on a water soluble component, a dicarboxylic acid functionalized with 4EG chains (WSCL) and two hydrophobic units, a metal containing component (cis-( $\left.\left.\mathrm{PEt}_{3}\right)_{2} \mathrm{Pt}(\mathrm{OTf})_{2}\right)(\mathrm{Pt}(\mathrm{II}))$ and tetra(4-pyridyphenyl)ethylene (TPPE) yielding the supramolecular complex Pt_12a, and tetrapyridylporphyrin (TPP) yielding the supramolecular complex Pt_12b (Figure 11).

Both complexes formed homogenous solutions in water and organic solvents. Complex Pt_12a exhibited a bright yellow fluorescence in polar solvents and blue luminescence in less polar solvents. In water, complex $\mathrm{Pt}$ _12a showed emission in the blue region of the spectra with increased intensity due to the formation of sheet-like structures. Hence, tuning of the shape and emission properties of nanostructures was obtained in $\mathrm{MeOH}$ solution with the addition of increasing amount of water. At low water content (10\%), $150 \mathrm{~nm}$ diameter nanoparticles were observed by SEM investigations that grew in diameter with increasing water content until $30 \%$. Between 40 and $70 \%$, the particles assembled into planes while sheet-like structures were totally formed at water content $>70 \%$. The sheet-like structure formation was accompanied by a drastic increase of the emission intensity and a blue-shift of the emission maxima.
Regarding complex Pt_12b, it was found that in water, it formed different shape aggregates as a function of concentration. By increasing the concentration, upon CAC which was found to be around $2.30 \cdot 10^{-6} \mathrm{M}$, multilayer wall vesicles with average diameter of ca. $200 \mathrm{~nm}$ and wall thickness $20 \mathrm{~nm}$ were formed that transformed into nanofibres at 5 . $10^{-4} \mathrm{M}$, due to the ordering of the hydrophobic cores through $\pi-\pi$ stacking while the hydrophilic 4EG chains extended radially from the cylindrical framework facing the water media. Although the emission spectra showed the characteristic bands of porphyrin, the intensity of the emission were lower with respect to those recorded in organic solvents, showing a quenching of fluorescence by aggregation.

4.2.2. Bulky Coordination Geometry Complexes. Although higher coordination number complexes have increased difficulty in ordering into soft supramolecular structures due to the bulky voluminous geometry, by a judicious functionalization remarkable self-assembling abilities were obtained also with octahedral $\operatorname{Re}(\mathrm{I}), \operatorname{Ru}(\mathrm{II})$, or $\operatorname{Ir}(\mathrm{III})$ species. The great efforts were compensated by the remarkable photophysical properties of these complexes in a biological relevant environment such as tunable emission color, intense fluorescence and emitting excited states generated by spin forbidden transitions. Importantly, the close-pack of these molecules into supramolecular assemblies may hinder the quenching phenomena due to dioxygen diffusion, a major drawback suffered by these complexes. On the other side, a close-pack of chromophores may lower the quantum yields and shorten the lifetimes due to formation of new excited states that may decay to the ground state via nonradiative paths. The 

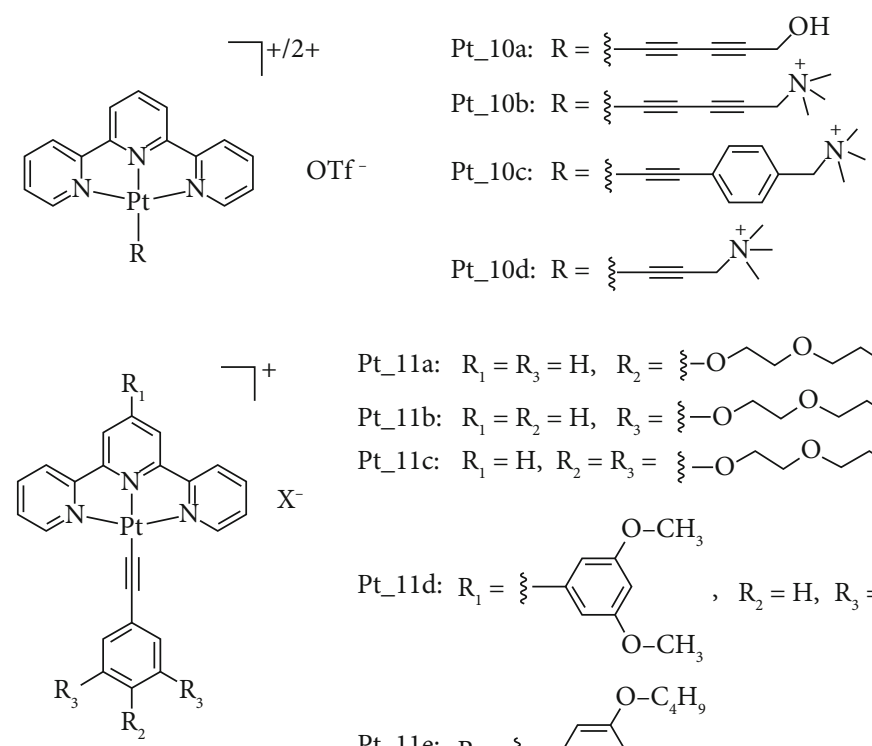

$\begin{array}{ll}\text { Pt_11a: } & \mathrm{R}_{1}=\mathrm{R}_{3}=\mathrm{H}, \mathrm{R}_{2}= \\ \text { Pt_11b: } & \mathrm{R}_{1}=\mathrm{R}_{2}=\mathrm{H}, \mathrm{R}_{3}=\xi-\mathrm{O} \\ \text { Pt_11c: } & \mathrm{R}_{1}=\mathrm{H}, \mathrm{R}_{2}=\mathrm{R}_{3}=\xi\end{array}$
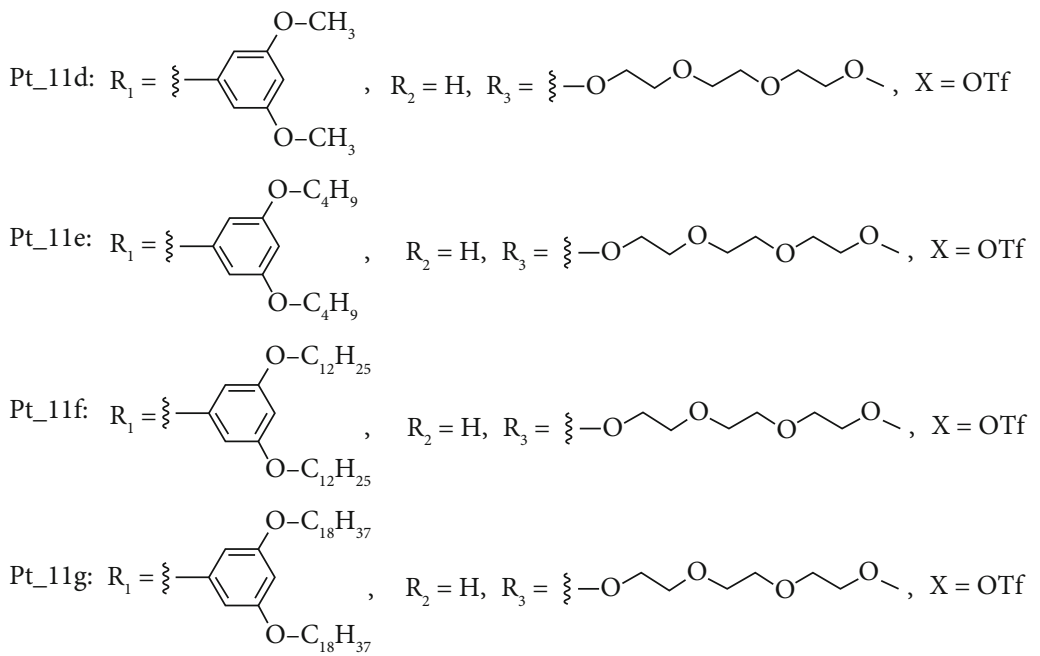

Figure 10: Amphiphilic Pt(II) complexes based on tpy and alkynyl ligands.

protophysical properties of these systems are thus usually resulting from a combination of different processes.

Hydrophobic dinuclear neutral $\operatorname{Re}(\mathrm{I})$ tricarbonyl complexes were functionalized with polar tails (3EG or 4EG) through rigid aromatic or flexible alkyl hydrophilic groups (complexes Re_1a-d in Figure 12) [116]. All complexes resulted soluble in dioxane, showing the photophysical features of monomeric species, respectively, featureless emission at r.t. arising mainly from ${ }^{3}$ MLCT excited state, centred at ca. $560 \mathrm{~nm}$ for complexes Re_1b-d, while the emission of Re_la is red-shifted to $603 \mathrm{~nm}$ due to the lower energy of its emitting excited state in line with a more $\pi$-conjugated ligand. In dioxane/water mixture, at high water content $(>80 \%)$, aggregation into globular supramolecular structures $\left(D_{h}=200-400 \mathrm{~nm}\right)$ with monodispersed nanosizes for $\mathrm{Re}_{-}$ $1 \mathrm{a}$ and $\mathrm{Re} \_1 \mathrm{c}$ and nonhomogeneous size distribution with the presence of small amorphous aggregates for complexes for Re_1b and Re_1d was observed. The aggregation was mainly attributed to the close packing of hydrophobic $\operatorname{Re}(\mathrm{I})$ complexes in the centre of the nanostructures with the hydrophilic tails forming a solvated shell. This was sustained by photophysical investigations, where a blue shift of the emission maxima with $15-30 \mathrm{~nm}$ was detected, meaning that the emitting state of the metal complexes experienced a less polar environment being protected from the direct contact with water. The aggregation was accompanied by a substan- tial increase of photoluminescence quantum yield and increased excited lifetime values which resulted monoexponential for complexes $\mathrm{Re} \_1 \mathrm{a}$ and $\mathrm{Re} \_1 \mathrm{c}$, respectively, biexponential for complexes Re_lb and Re_1d. Complexes Re_1b and Re_ld having longer connecting units with higher mobility formed less uniform aggregates, while complex Re_1c with reduced overall hydrophobicity aggregated at higher water content (90\%), however, forming larger supramolecular architectures with the highest values of emission quantum yields $(\Phi=0.19)$. The best packing capability was shown by complex Re_la, where the extra phenyl group probably favoured additional intermolecular stabilization through $\pi-\pi$ interactions.

The self-assembly of alkoxy-bridged dinuclear $\operatorname{Re}(\mathrm{I})$ (complexes Re_2a-c in Figure 12) molecular rectangles into nanosized aggregates was studied in ACN-water mixtures by Manimaran et al. [117]. The formation of aggregates with increasing water content was accompanied by a substantial increase of the emission intensity and lifetimes, and a blueshift of the emission maxima, indicating a situation in which the complexes suffer by a lesser extent from exposure to solvent molecules, experiencing a more rigid environment and a less distorted excited-state environment. DSL experiments supported the presence of aggregation; however, no further information about the morphology of the nanostructures was given. 


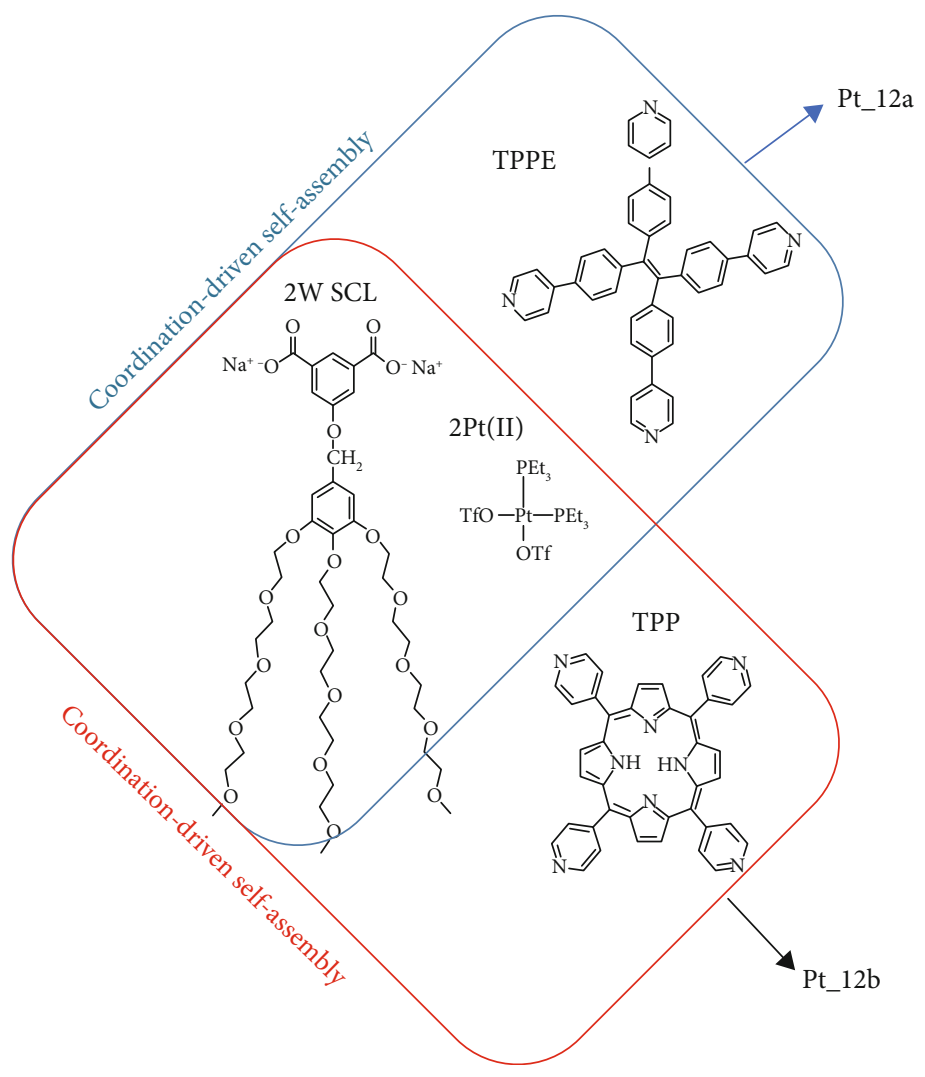

Figure 11: Chemical structures of the different units forming the supramolecular metallaclips Pt_12a and Pt_12b.

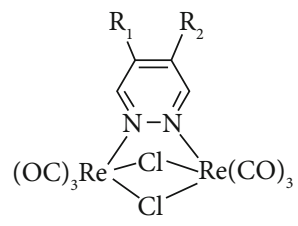

$$
\begin{array}{lll}
\text { Re_la: } & \mathrm{R}_{1}=\mathrm{H} & \mathrm{R}_{2}= \\
\mathrm{Re} \_1 \mathrm{~b}: & \mathrm{R}_{1}=\mathrm{CH}_{3} & \mathrm{R}_{2}=-\left(\mathrm{CH}_{2}\right)_{8} \mathrm{O}\left(\mathrm{CH}_{2} \mathrm{CH}_{2} \mathrm{O}\right)_{3} \mathrm{CH}_{3} \\
\text { Re_1c: } & \mathrm{R}_{1}=\mathrm{C}_{2} \mathrm{H}_{5} & \mathrm{R}_{2}=-\left(\mathrm{CH}_{2}\right)_{2} \mathrm{O}\left(\mathrm{CH}_{2} \mathrm{CH}_{2} \mathrm{O}\right)_{3} \mathrm{CH}_{3} \\
\text { Re_ld: } & \mathrm{R}_{1}=\mathrm{CH}_{3} & \mathrm{R}_{2}=-\left(\mathrm{CH}_{2}\right)_{8} \mathrm{O}\left(\mathrm{CH}_{2} \mathrm{CH}_{2} \mathrm{O}\right)_{4} \mathrm{CH}_{3}
\end{array}
$$$$
\text { 每 }
$$$$
\text { Re_2a: } \quad \mathrm{R}=-\left(\mathrm{CH}_{2}\right)_{3} \mathrm{CH}_{3}
$$$$
\text { Re_2b: } \quad \mathrm{R}=-\left(\mathrm{CH}_{2}\right)_{7} \mathrm{CH}_{3}
$$$$
\mathrm{Re} \_2 \mathrm{c}: \quad \mathrm{R}=-\left(\mathrm{CH}_{2}\right)_{11} \mathrm{CH}_{3}
$$

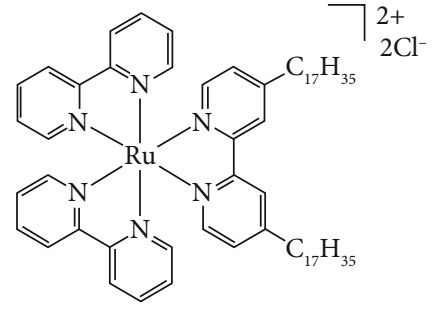

$\mathrm{Ru} \_1$

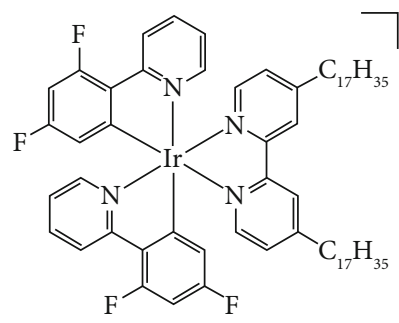

Ir_1<smiles>NS(=O)(=O)Oc1cccn(P(c2ccccc2)(c2ccccc2-c2ccccc2)(c2ccccc2-c2ccccn2)[n+]2ccccc2)c1=O</smiles>

Ir_2a: $\quad \mathrm{R}=-\mathrm{C}_{6} \mathrm{H}_{12}{ }^{-}$

Ir_2b: $\mathrm{R}=-\mathrm{C}_{12} \mathrm{H}_{24}-$

FIgURE 12: Neutral and ionic octahedral metal complexes. 
Amphiphilic ionic complexes Ru_1 and Ir_1 (Figure 12), having the charged complex cation as hydrophilic unit and one of the bipyridine ligands functionalized in the $4,4^{\prime}$-positions with 17 methylene units as hydrophobic part, similar to classical surfactants, were reported [118]. Upon CAC $\left(5 \cdot 10^{-5} \mathrm{M}\right.$ for complex Ru_1 and $\left.3 \cdot 10^{-6} \mu \mathrm{M}\right)$, the complexes aggregated into spherical micelles formed by the segregation of the hydrophobic chains inside the core, with the charged hydrophilic complex cations facing the water media. The formation of the aggregates was sustained by the appearance of an additional longer emission lifetime and the increase of emission quantum yields, indicative of a close proximity of chromophores that reduced the nonradiative decays due to vibrational modes and disfavoured oxygen diffusion. Mixed micellar structures were obtained with efficient electronic energy-transfer processes between the electronic energy acceptor Ru_1 and the energy donor Ir_1.

A reverse molecular design was further employed: the molecular amphiphiles were formed by a neutral emissive Ir(III) complex with two cyclometallating phenylpyridine ligands and a picolinate ancillary ligand substituted with long alkyl chains ending with charged solubilizing sulfate groups (complexes Ir_2a-b in Figure 12) [119]. Aggregation into vesicular spherical aggregates, with the hydrophobic $\operatorname{Ir}(\mathrm{III})$ complexes segregated from water and the charged sulfate chains facing the solvent was obtained for both species at concentrations above $10^{-5} \mathrm{M}$. The particles had averaged hydrodynamic radii $\left(\mathrm{R}_{\mathrm{h}}\right)$ of ca. $1340 \pm 707$ and $202 \pm 159 \mathrm{~nm}$ at $10^{-3} \mathrm{M}$, for complexes Ir_2a and Ir_2b, respectively, with different emission profiles despite the similarity of the chemical structures. Indeed, a blue-shift of the emission maxima with respect to low concentrated solutions was observed for both complexes upon aggregation, complex Ir_2a showing a structured emission with maxima at 509 and $531 \mathrm{~nm}$, while complex Ir_2b exhibited emission centred at $543 \mathrm{~nm}$, with a noticeable prolongation of the excited-state lifetimes of both systems, despite the presence of dioxygen in the aqueous media. Thus, the increase in the length of the alkyl chain for complex Ir $2 \mathrm{~b}$ yielded more soft and flexible aggregates, with longer emission wavelength and faster radiative kinetics.

\subsection{Nonconventional Structure Transition Metal} Coordination Complexes. Some examples of luminescent metal complexes that do not have well-defined polar/apolar molecular parts but self-assembly through similar mechanisms and forces into supramolecular structures in aqueous media were quite recently reported. This included an $\mathrm{Au}(\mathrm{I})$ linear complex formed with two hydrophilic monodentate ligands and some $\mathrm{Pt}(\mathrm{II})$ and $\operatorname{Ir}(\mathrm{III})$ complexes that selfassembled into chromonic or chromonic-type lyotropic liquid crystalline states.

The linearly coordinated Au_1 complex (Figure 13) formed with a water soluble trisulfonated-triphenylphosphane, and a hydrophilic peptide was shown to form welldefined spherical nanoparticles in buffered water solution under physiological ionic strength [120]. The micellar solutions at a concentration of $66.7 \mu \mathrm{M}$ showed an unstructured weak long-lived emission band centred at $520 \mathrm{~nm}$ from trip-

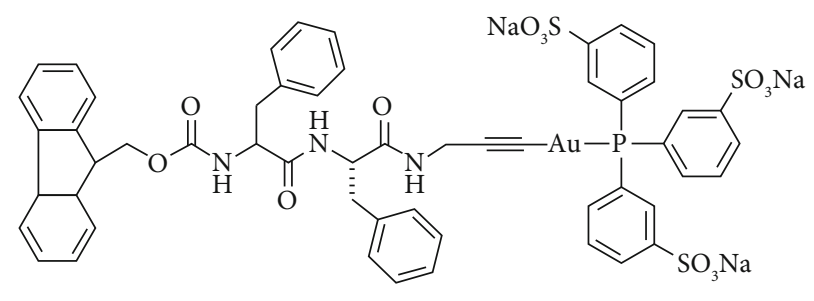

Au_1

FIgURE 13: Linear amphiphilic Au(I) complex based on peptide unit and trisulfonated-triphenylphosphane ligand.

let states, indicative of $\mathrm{Au} \cdots \mathrm{Au}$ metallophilic interactions. Increasing the ionic strength of the solution by adding $\mathrm{NaCl}$, the intensity of the emission increased without affecting the emission energy or the excited state lifetimes. This pointed towards a stabilization of the nanostructures due to the screening of the repulsive Coulombic interactions and the increase of hydrophobic effect, without affecting the morphology of the aggregates. However, without $\mathrm{NaCl}$, large densely packed sheet-like structures were formed, accompanied by a red-shift of the luminescence emission maxima due to a more reduced $\mathrm{Au} \cdots \mathrm{Au}$ distance in the planar morphology.

\subsubsection{Chromonic or Chromonic-Like Coordination Complexes}

(1)Pt(II) and Rh(I) Square-Planar Complexes. Transition metal coordination complexes with surfactant-like amphiphilic structures have principally the requisites to form lyotropic liquid crystalline structures with increasing concentration. However, no report regarding such mesomorphism was mentioned, most probably due to the lower solubility of such species with respect to the concentration needed for ordering. Recently, some coordination complexes that do not have the classical amphiphilic structure, respectively flexible hydrophobic or hydrophilic chains, were reported to form nanostructures in water, mostly due to metallophilic, aromatic stacking, and H-bonding interactions. A contribution of hydrophobic/hydrophilic interactions due to differences in the character of the molecular units, however, cannot be excluded. In some cases, when anisotropic shape structures are formed, these assemble further into ordered lyotropic chromonic or chromonic-like liquid crystalline states. In contrast to the well-studied surfactant systems, lyotropic chromonics are still poorly understood [121-123]. The supramolecular structures, ordering, and self-assembling mechanisms still need appreciable efforts to be understood. Work dedicated to the synthesis of luminescent transition metal complexes able to form ordered chromonic lyotropic structures in water may bring important information in elucidating its mechanism of self-assembly and mesophase morphology, besides the obvious appealing practical applications for bioimaging or biosensing. However, it is quite challenging to retain the luminescence properties in water media, due to aggregation induced quenching processes through oxygen diffusion or 

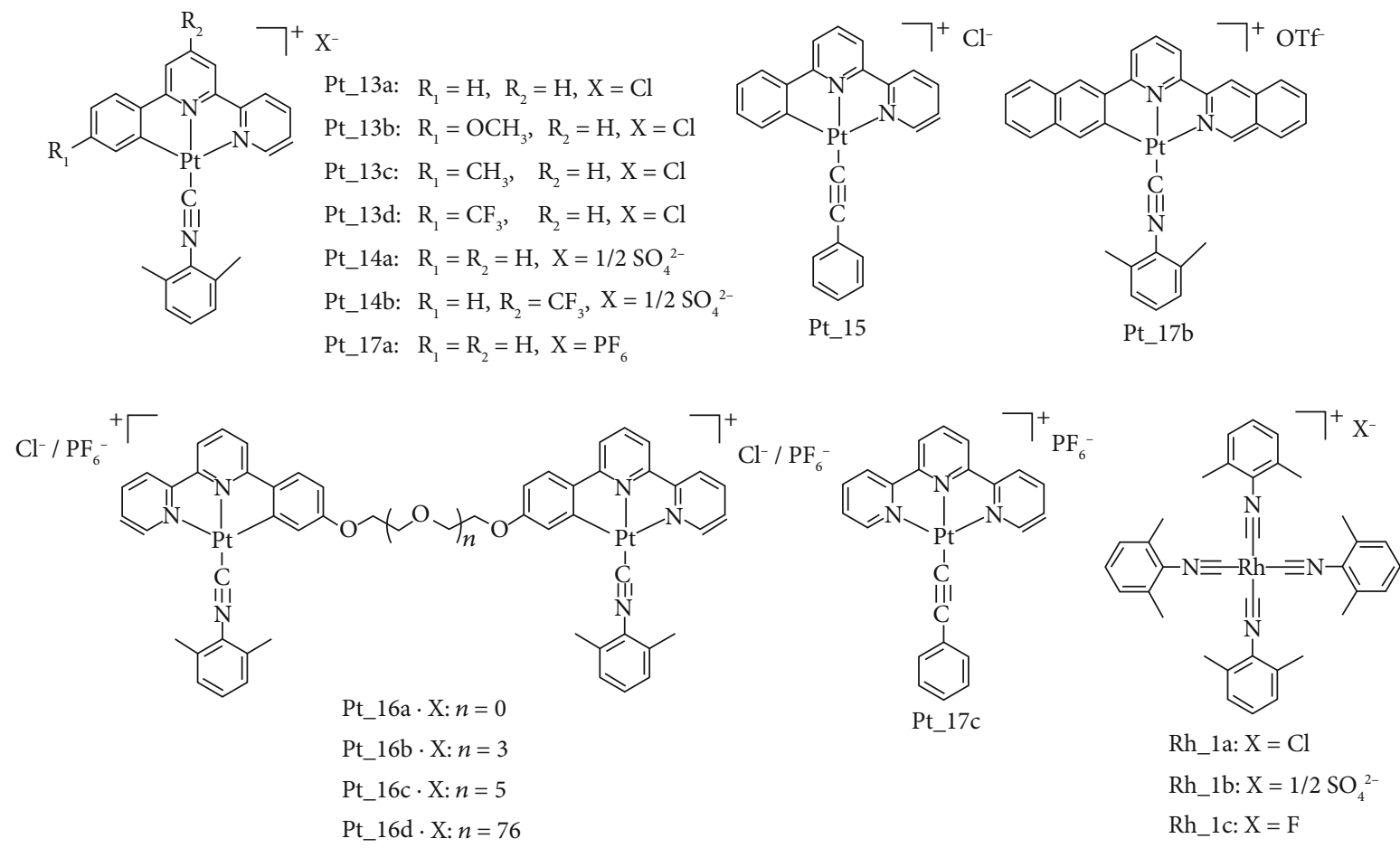

Figure 14: Planar-shape cationic Pt(II) and $\mathrm{Rh}(\mathrm{I})$ complexes.

aggregation causing radiationless decay in solution. Studies reporting on linear $\mathrm{Au}(\mathrm{I})$ [124] and tetracoordinated $\mathrm{Ag}(\mathrm{I})$ [125] showed no emission properties in water probably due to a total quenching of luminescence.

In this ambit, Lu et al. reported luminescent planar-shape cationic Pt(II) complexes having solubilizing chloride or sulfate anions, able to self-assemble in water into ordered polyelectrolitic microfibres (complexes Pt_13a-d, Pt_14a-b, and Pt_15 in Figure 14) [126]. The complexes formed oligomeric structures even at very low concentration in water (ca. 0.0013 wt\%). Unsubstituted complexes (Pt_13a, Pt_14a, and $\mathrm{Pt}$ 15) exhibited in water a structureless red emission centred at 677 (Pt_14a) and $655 \mathrm{~nm}\left(\mathrm{Pt} \_15 \mathrm{a}\right)$ assigned to a ${ }^{3} \mathrm{MMLCT}$ excited state due to the formation of molecular aggregates through Pt...Pt interactions, while complex Pt_15 was not emissive. The formation of oligomers was followed by addition of increased volumes of water into a methanolic solution of complexes $\mathrm{Pt}$ _13a and $\mathrm{Pt} \_$14a where the complexes exist as solvated single molecules, exhibiting vibronically structured high-energy emissions with peak maxima at 525 and $480 \mathrm{~nm}$ assigned to a mixture of ${ }^{3} \mathrm{MLCT} /{ }^{3} \mathrm{ILCT}$ (intraligand charge transfer) excited states. With increasing water ratio, the formation of oligomers through metallophilic interactions is clearly evidenced by the substantial increase of emission energy and red-shift of the emission maxima. Interestingly, further increase of complex concentration in water leads to the formation of lyotropic chromonic liquid crystalline phases, as proven by POM observations and ${ }^{1} \mathrm{H}$ and ${ }^{2} \mathrm{H}$ NMR studies. The concentration for the mesophase formation depended strongly on the ligands' and substituents' nature, being estimated at r.t. around 1.5, 2.0, and
$10 \mathrm{wt} \%$ for complexes Pt_13a-c, Pt_14a-b, and Pt_15, respectively. The mesophases of complexes $\mathrm{Pt} \_13 \mathrm{a}-\mathrm{c}$ and $\mathrm{Pt} \_14 \mathrm{a}-\mathrm{b}$ showed exceptionally high extensional viscosity, permitting the obtainment of semicrystalline, optically uniaxial, and strongly luminescent microfibres directly from concentrated solutions. Metallophilic $\mathrm{d}^{8} \cdots \mathrm{d}^{8}$ and ligand-ligand interactions were observed along the fibres' long axis that together with hydrophobic interactions overrode the electrostatic repulsion between charged species, permitting the cations to pile up and form polyelectrolytic microfibres which further organize in water into chromonic ordered mesophases.

Complex Pt_13a was furthermore covalently interconnected through a flexible oligo(oxyethylene) chain of different length obtaining, thus, dicationic species Pt_16a-d.X (Figure 14) that as a function of the bridge length formed hydrogels with optical anisotropy and 1D nematic order deriving from the original complex [127]. The mesophase morphology and luminescence was modulated by the interand intramolecular interactions dictated by the oligo(oxyethylene) flexible bridge. Indeed, the emission energies of the complexes $\mathrm{Pt}$ _16a-d.X recorded in ACN solution at r.t. are remarkably different, revealing a significant influence of the intra- and/or intermolecular interactions, the spectroscopic behaviour in temperature showed a predominance of intermolecular interactions for complexes Pt_16b.PF6 and Pt_16c.PF6 with moderate bridge length. In water solution, even at low concentration $\left(10^{-5} \mathrm{M}\right)$, a red-shifted emission for complexes containing $\mathrm{Cl}$ as counterion $\mathrm{Pt}$. $16 \mathrm{a}-\mathrm{d} \cdot \mathrm{Cl}$ was observed due to oligomerization through $\mathrm{Pt} \ldots \mathrm{Pt}$ and $\pi-\pi$ stacking interactions, while with increasing concentration, liquid crystalline phases were formed. Complexes $\mathrm{Pt} \_16 \mathrm{~b} \cdot \mathrm{Cl}$ and $\mathrm{Pt} \_16 \mathrm{c} \cdot \mathrm{Cl}$ formed liquid crystalline phases in water at 
2 wt $\%$ and hydrogels which still maintained the liquid crystalline ordering at higher concentration $(4.8 \mathrm{wt} \%)$, while the mesophase of complex Pt_16d.Cl retained its fluidity even at high complex concentration (>15 wt\%).

Planar shape $\mathrm{Rh}(\mathrm{I})$ coordination complexes based on 2,6-xylylisocyanide ligands bearing solubilizing counterions (complexes Rh_1a-c in Figure 14) were showed to selfaggregate into luminescent crystalline nanowires through a nucleation-elongation mechanism, distinct from the isodesmic aggregation of chromonic compounds [128]. As a function of concentration and processing, the complexes formed a variety of hierarchically organized superstructures: by solubilizing the complexes at low concentration in boiling water $\left(10^{-5}-10^{-4} \mathrm{M}\right)$, dimeric aggregates with short $\mathrm{Rh} \cdots \mathrm{Rh}$ interactions formed, while by cooling the red-wine solutions, additional molecules associated to the dimers, the color changed to blue, typical for trimeric or higher number aggregates. After aging the solutions at r.t., randomly distributed ultralong crystalline nanowires in the blue aqueous were obtained, with a structureless NIR emission centred at $806 \mathrm{~nm}$, typical to extended $\mathrm{RhI} \cdots \mathrm{RhI}$ interactions.

(2)Ir(III) Octahedral Complexes. Although chromonic systems are generally characterized by planar structures surrounded by peripheral solubilizing and/or ionic groups, or ionic square planar geometry metal complexes having hydrophilic counterions, Yadav et al. showed for the first time self-assembly into chromonic-like mesophases of bulky octahedral luminescent Ir(III) complexes [129] (complexes Ir_3a-c in Figure 15). Even if they belong to a family of $\operatorname{Ir}(\mathrm{III})$ emitters of general formula $\left[(p p y)_{2} \operatorname{Ir}\left(\mathrm{N}^{\wedge} \mathrm{N}\right)\right]^{+} \mathrm{X}^{-}$where $p p y$ is 2-phenylpyridine and $\mathrm{N}^{\wedge} \mathrm{N}$ is $2,2^{\prime}$-bipyridine or 1,10-phenanthroline, highly researched for practical for applications in Light Emitting Electrochemical Cells (LEECs) [130, 131], only by changing the counterions with carboxylates of various alkyl chains, water solubility, and self-assembling ability into chromoniclike liquid crystalline phases was induced [132] (complexes Ir_4 in Figure 15).

A detailed structural analysis by WAXS, SAXS, and SANS experiments performed for Ir_3a complex in both anisotropic gel and isotropic phases showed that in water the complexes self-assembled into double string polyelectrolytic supramolecular columns surrounded by solvated counterions. These aggregated species existed also at low concentration $(1 \% w / w)$ but without positional order, and grew unidimensionally by increasing concentration until the transition into $2 \mathrm{D}$ rectangular lattices $(1.3-1.8 \% w / w$ for complexes Ir_3a-c and 6.0\% $w / w$ for complex Ir_4), accompanied by a drastic increase of viscosity.

The gelling and appearance of positional long-range order specific to liquid crystalline systems resulted only from the interactions between increasing number of strands, with preserving their shape, similar to the assembly of chromonic polyelectrolyte columns. Due to structural differences between the typical chromonic planar molecules and the Ir(III) complexes with rather bowl-like geometry, the self-

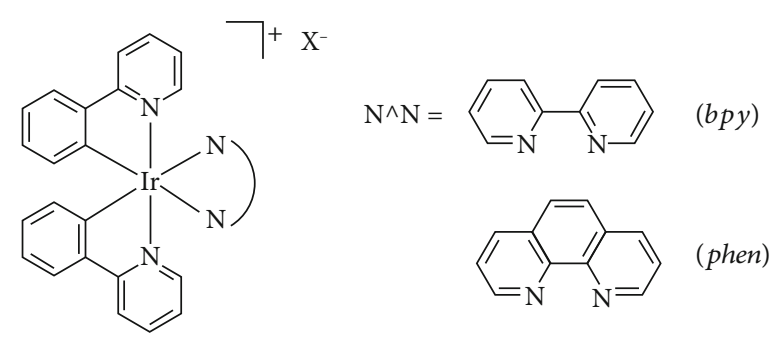

$\begin{array}{ll}\text { Ir_3a: } & \mathrm{N}^{\wedge} \mathrm{N}=b p y, \quad \mathrm{X}=\mathrm{CH}_{3} \mathrm{COO} \\ \text { Ir_3b: } & \mathrm{N}^{\wedge} \mathrm{N}=b p y, \quad \mathrm{X}=\mathrm{C}_{2} \mathrm{H}_{7} \mathrm{COO} \\ \text { Ir_3c: } & \mathrm{N}^{\wedge} \mathrm{N}=b p y, \quad \mathrm{X}=\mathrm{C}_{5} \mathrm{H}_{11} \mathrm{COO} \\ \text { Ir_4: } & \mathrm{N}^{\wedge} \mathrm{N}=\text { phen, } \quad \mathrm{X}=\mathrm{CH}_{3} \mathrm{COO}\end{array}$

FIgURE 15: Octahedral cationic Ir(III) complexes.

assembly was tentatively explained by a difference in the charge distribution in the coordination shell forming areas with rather hydrophobic character next to areas with rather hydrophilic character that led to aggregation in water.

Accurate photophysical investigations carried out on isotropic water solutions containing the polyelectrolytic strings without positional order and viscous mesophases at room temperature by varying temperature sustained the structural investigations and offered more information about the system behaviour, considering a similar aggregation for all complexes Ir_3a-c and Ir_4. Importantly, a significant improvement of the emission efficiencies and blue-shift of the emission maxima was observed in water with respect to the solvated complexes in diluted methanol. Due to aggregation, the enhancement of rigidity and lack of strong interchromophoric contacts experienced by the $\operatorname{Ir}(\mathrm{III}) \quad b p y$-based complexes Ir_3a-c, the isotropic aqueous solutions $(1 \% \mathrm{w} / \mathrm{w})$ at r.t. shows emission mainly from a ${ }^{3}$ MLCT state, with a progressive blue-shift of the emission maxima with increasing counterion chain length $(\lambda=564,554$, and $540 \mathrm{~nm}$ for complexes Ir_3a, Ir_3b, and Ir_3c, respectively) due to an increase of medium rigidity. The triexponential emission decay recorded suggested several different molecular environment experienced by the Ir(III) complexes. Thus, the longest luminescent lifetime component was attributed to the fraction of $\operatorname{Ir}(\mathrm{III})$ molecules forming the strand structures, while the shorter lifetime minor fraction component was attributed to the $\operatorname{Ir}(\mathrm{III})$ species in molecular form or existing as smaller aggregates. Keeping the concentration constant $(1 \% w / w)$, the isotropic solution of complex Ir_3c already contained an important fraction of the longer time component $(81.5 \%)$ with respect to its shorter alkyl chain analogues Ir_3a (43\%) and Ir_3b (50\%) indicating a lowering of gelation concentration.

A substantial increase of the number of $\operatorname{Ir}(\mathrm{III})$ molecules self-associating to form strands for complexes $\operatorname{Ir}_{-} 3 \mathrm{a}$ and $\mathrm{Ir}_{-}$ $3 \mathrm{~b}$, with complete (Ir_3a and Ir_3c) or quasicomplete disappearance (Ir_3b) of the monomeric species was found in the mesophases $(2.5 \% w / w)$. By increasing temperature, a significant decrease of the luminescence lifetimes and emission efficiencies is observed, accompanied by a negligible red- 
shift of the emission maxima, indicating different quenching mechanisms beside the nonradiative processes related to thermal agitation, respectively, a more efficient oxygen quenching process due to the increase of oxygen mobility.

By changing the ancillary ligand from bpy to phen, increasing the rigidity and aromaticity of the ligand, a higher concentration is needed for the transition to the viscous mesophase $(6.0 \%)$ for complex Ir_ 4 with respect to complex Ir_3a having both acetate as counterions, while a lower viscosity and more fluid mesophase is obtained for the former. Accurate rheological investigations carried out on the isotropic and mesomorphic water solutions of complexes Ir_3a and its analogues Ir_4 corroborated with photophysical data showed important differences in their selfassembling ability. Regarding complex Ir_4, photophysical investigations carried out in water diluted solution (1\% $w / w)$ showed a biexponential life time, with an emission maxima centred at $600 \mathrm{~nm}$ and an emission efficiency of $0.06 \%$. Increasing concentration to $6.5 \% w / w$, a blue-shift of the emission maxima $(\lambda=570 \mathrm{~nm})$ and a multiexponential emission decay were observed, which were attributed to aggregation phenomena. The weight of the components is distributed between two types of aggregates: polyelectrolytic strands and smaller aggregates with more symmetric shape, thus forming a less viscous and more dynamic ordered mesophase evidenced by rheological investigations.

\section{Concluding Remarks and Perspectives}

Supramolecular self-assembly is a key approach for the design and development of nanostructured systems and has become a fundamental method for the formation of advanced nanomaterials. This strategy focuses on different types of interacting (and communicating) building blocks to perform preprogrammed advanced functions [133-136]. The novel structures and properties obtained by the efficient use of noncovalent forces (and structure directing interactions) provide additional flexibility for the design and development of versatile smart materials [137-139], biomaterials [140-142], and functional nanodevices [143-145].

The self-assembly processes may become particularly intriguing if amphiphilic molecules are involved. In addition to the wide scenario of possible interactions between amphiphiles (polar and apolar interactions, steric hindrance, H-bonds, $\pi-\pi$ interactions, etc.), further situations have been considered in this work: (i) the existence of multiple polar and apolar groups within the molecular architecture and (ii) the presence of metal atoms, which can directly interact with the amphiphiles by coordination bonds and with themselves by metallophilic bonds. The consequent formation of amphiphilic metal complexes causes a real explosion of the number of possible supramolecular structures. In this context, if noble metal atoms are involved, novel photophysical properties can be in parallel envisaged. $\mathrm{Pt}(\mathrm{II})$ certainly plays a pivotal role due to its versatility in forming supramolecular structures, but also $\mathrm{Re}, \mathrm{Rh}, \mathrm{Ru}, \mathrm{Ir}$, and $\mathrm{Au}$ give interesting photophysical properties within certain complexes. Type of metal, oxidation state, counterions, number of complexing sites in the ligands, and their amphiphilicity (distribution of polar and apolar parts within their molecular architecture) as well as concentration, type of solvent, and temperature are all factors governing the overall self-assembly and, consequently the behaviour towards the absorption of photons of the resulting structures, the nature of the excited states, and the modes of decays. Modern research is obviously exploring the possibility to exploit all these potentialities for specific applications (bioimaging, biosensing, Light Emitting Electrochemical Cells, etc.). However, in our opinion, these potentialities are even more effective if molecular architecture can be manipulated by synthetic chemistry, allowing the obtainment of a virtually unlimited scenario of possible molecules and consequently novel properties. The rich ingredients we have shown in this work allow researchers to push towards the invention of smart materials, i.e., those materials whose photophysical properties can change according to an external stimulus. After all, this path has already been seen in living structures, where the availability of a high number of chemical species, together with the intelligent use of opportune molecules, causes the formation of complex structures with emerging properties and complex behaviour, able to react to an external stimulus.

In a similar way, the possibility to control the complexity in the nanoworld would make real the building up of "artificial" systems that could improve human life by realizing novel complex molecular materials/systems/devices amplifying the range of their capabilities in every desired field. We really hope, with this contribution, to have made the reader curious to this exciting aspect, encouraging, at the same time, future research to focus on this aspect. A gradual development is advisable, passing from the exploitation of the individual/specific properties of the compounds we have presented, to the tuning of their properties in accordance with suitable external stimuli. This is to give, hopefully, stimuli-responsive smart materials, which, for sure, represent one of the most appealing trends in current scientific research.

\section{Data Availability}

The data used to support the findings of this study are included within the article.

\section{Conflicts of Interest}

The authors declare that there is no conflict of interest regarding the publication of this paper.

\section{Acknowledgments}

E. I. S. and C. C. acknowledge the support of the Romanian Academy, Project 4.1. The authors also acknowledge the support from the Romanian Academy and from the CNRRA bilateral project 2020-2022 (prot. n. 0088276 from 09/12/2019). 


\section{References}

[1] L. J. Chen and H. B. Yang, "Construction of stimuliresponsive functional materials via hierarchical selfassembly involving coordination interactions," Accounts of Chemical Research, vol. 51, no. 11, pp. 2699-2710, 2018.

[2] A. J. Moro, A. M. Pana, L. Cseh et al., "Chemistry and photochemistry of 2,6-Bis(2-hydroxybenzilidene)cyclohexanone. An example of a compound following the anthocyanins network of chemical reactions," The Journal of Physical Chemistry A, vol. 118, no. 32, pp. 6208-6215, 2014.

[3] M. Ramanathan, L. K. Shrestha, T. Mori, Q. Ji, J. P. Hill, and K. Ariga, "Amphiphile nanoarchitectonics: from basic physical chemistry to advanced applications," Physical Chemistry Chemical Physics, vol. 15, no. 26, pp. 1058010611, 2013.

[4] M. H. Yen, J. Chaiprapa, X. Zeng et al., “Added alkane allows thermal thinning of supramolecular columns by forming superlattice-an X-ray and neutron study," Journal of the American Chemical Society, vol. 138, no. 18, pp. 5757-5760, 2016.

[5] D. Lombardo, P. Calandra, S. Magazù et al., "Soft nanoparticles charge expression within lipid membranes: the case of amino terminated dendrimers in bilayers vesicles," Colloids and Surfaces B: Biointerfaces, vol. 170, pp. 609616, 2018.

[6] X. Yao, L. Cseh, X. Zeng, M. Xue, Y. Liu, and G. Ungar, "Body-centred cubic packing of spheres - the ultimate thermotropic assembly mode for highly divergent dendrons," Nanoscale Horizons, vol. 2, no. 1, pp. 43-49, 2017.

[7] D. Lombardo, P. Calandra, L. Pasqua, and S. Magazù, "Selfassembly of organic nanomaterials and biomaterials: the bottom-up approach for functional nanostructures formation and advanced applications," Materials, vol. 13, no. 5, p. 1048, 2020.

[8] B. R. Cooper, H. Ehrenreich, and H. R. Philipp, "Optical properties of noble metals. II," Physical Review, vol. 138, no. 2A, pp. A494-A507, 1965.

[9] R. L. Jacobs, "The electronic structure of the noble metals I. the energy bands," Journal of Physics C: Solid State Physics, vol. 1, no. 5, pp. 1296-1306, 1968.

[10] P. Calandra, D. Caschera, V. Turco Liveri, and D. Lombardo, "How self-assembly of amphiphilic molecules can generate complexity in the nanoscale," Colloids and Surfaces A: Physicochemical and Engineering Aspects, vol. 484, pp. 164-183, 2015.

[11] S. He, C. Li, Q. Zhang et al., "Tailoring platinum(IV) amphiphiles for self-targeting all-in-one assemblies as precise multimodal theranostic nanomedicine," ACS Nano, vol. 12, no. 7, pp. 7272-7281, 2018.

[12] H. Xiao, G. T. Noble, J. F. Stefanick et al., "Photosensitive $\mathrm{Pt}(\mathrm{IV})$-azide prodrug-loaded nanoparticles exhibit controlled drug release and enhanced efficacy in vivo," Journal of Controlled Release, vol. 173, pp. 11-17, 2014.

[13] H. K. Cheng, C. Y. S. Chung, K. Zhang, and V. W. W. Yam, "Simple and versatile preparation of luminescent amphiphilic platinum(II)-containing polystyrene complexes with transformable nanostructures assisted by Pt...Pt and $\pi-\pi$ interactions," Chemistry: An Asian Journal, vol. 12, no. 13, pp. 1509-1516, 2017.

[14] X. Yan, T. R. Cook, P. Wang, F. Huang, and P. J. Stang, "Highly emissive platinum(II) metallacages," Nature Chemistry, vol. 7, no. 4, pp. 342-348, 2015.
[15] M. Panigati, M. Mauro, D. Donghi et al., "Luminescent dinuclear rhenium(I) complexes containing bridging 1,2diazine ligands: photophysical properties and application," Coordination Chemistry Reviews, vol. 256, no. 15-16, pp. 1621-1643, 2012.

[16] M. Y. Leung, S. Y. L. Leung, K. C. Yim, A. K. W. Chan, M. Ng, and V. W. W. Yam, "Multiresponsive luminescent cationic cyclometalated gold(III) Amphiphiles and their supramolecular assembly," Journal of the American Chemical Society, vol. 141, no. 49, pp. 19466-19478, 2019.

[17] K. M. C. Wong and V. W. W. Yam, "Self-assembly of luminescent alkynylplatinum(II) terpyridyl complexes: modulation of photophysical properties through aggregation behavior," Accounts of Chemical Research, vol. 44, no. 6, pp. 424-434, 2011.

[18] I. Hamley, Introduction to Soft Matter, Wiley, Chichester, 2000.

[19] H. Hu, M. Gopinadhan, and C. O. Osuji, "Directed selfassembly of block copolymers: a tutorial review of strategies for enabling nanotechnology with soft matter," Soft Matter, vol. 10, no. 22, pp. 3867-3889, 2014

[20] M. Shimomura and T. Sawadaishi, "Bottom-up strategy of materials fabrication: a new trend in nanotechnology of soft materials," Current Opinion in Colloid \& Interface Science, vol. 6, no. 1, pp. 11-16, 2001.

[21] R. A. L. Jones, Soft Condensed Matter, vol. 23, no. 6, 2002, Oxford University Press, UK, 2002.

[22] R. Backova, "Combining soft matter and soft chemistry: integrative chemistry towards designing novel and complex multiscale architectures," Soft Matter, vol. 2, no. 6, pp. 452-464, 2006.

[23] V. T. Liveri, D. Lombardo, M. Pochylski, and P. Calandra, "Molecular association of small amphiphiles: origin of ionic liquid properties in dibutyl phosphate/propylamine binary mixtures," Journal of Molecular Liquids, vol. 263, pp. 274281, 2018.

[24] J. M. Guevara-Vela, E. Romero-Montalvo, V. A. Mora Gómez et al., "Hydrogen bond cooperativity and anticooperativity within the water hexamer," Physical Chemistry Chemical Physics, vol. 18, no. 29, pp. 19557-19566, 2016.

[25] G. M. Whitesides and D. J. Lipomi, "Soft nanotechnology: "structure"vs."function"," Faraday Discussions, vol. 143, pp. 373-384, 2009.

[26] D. Lombardo, G. Munaò, P. Calandra, L. Pasqua, and M. T. Caccamo, "Evidence of pre-micellar aggregates in aqueous solution of amphiphilic PDMS-PEO block copolymer," Physical Chemistry Chemical Physics, vol. 21, no. 22, pp. 1198311991, 2019.

[27] C. N. Likos, "Effective interactions in soft condensed matter physics," Physics Reports, vol. 348, no. 4-5, pp. 267-439, 2001.

[28] M. Li, Z. Luo, and Y. Zhao, "Self-assembled hybrid nanostructures: versatile multifunctional nanoplatforms for cancer diagnosis and therapy," Chemistry of Materials, vol. 30, no. 1, pp. 25-53, 2017.

[29] Y. Wang, H. Xu, and X. Zhang, "Tuning the amphiphilicity of building blocks: controlled self-assembly and disassembly for functional supramolecular materials," Advanced Materials, vol. 21, no. 28, pp. 2849-2864, 2009.

[30] D. Demus, J. W. Goodby, G. W. Gray, H. W. Spiess, and V. Vill, Handbook of Liquid Crystals, Wiley-VCH, Weinheim, 1998. 
[31] Y. Chen, P. Ma, and S. Gui, "Cubic and hexagonal liquid crystals as drug delivery systems," BioMed Research International, vol. 2014, Article ID 815981, 12 pages, 2014.

[32] R. Zana, Dynamics of Surfactant Self-Assemblies: Micelles, Microemulsions, Vesicles and Lyotropic Phases, R. Zana, Ed., Taylor \& Francis, London, 2005.

[33] V. De Giorgio and M. Corti, Physics of Amphiphiles: Micelles, North-Holland, Vesicles and Microemulsions, 1985.

[34] F. Evans and H. Wennerström, The Colloidal Domain, Wiley, New York, NY, USA, 2nd ed. edition, 1999.

[35] J. N. Israelachvili, "Thermodynamic and geometric aspects of amphiphile aggregation into micelles, vesicles and bilayers, and the interactions between them," in Proceedings in Physics of Amphiphiles: Micelles, Vesicles and Microemulsions, V. Degiorgio and M. Corti, Eds., pp. 24-58, North-Holland, Amsterdam, Netherlands, 1985.

[36] J. N. Israelachvili, Intermolecular and Surface Forces, Academic Press, New York, NY, USA, 2nd edition edition, 1992.

[37] J. Katsaras and T. Gutberlet, Lipid Bilayers Structure and Interactions, Springer, Berlin, Germany, 2001.

[38] J. M. Seddon and R. H. Templer, "Polymorphism of lipidwater systems," in in Handbook of Biological Physics, R. Lipowsky and E. Sackmann, Eds., pp. 97-160, Elsevier, 1995.

[39] M. Kotlarchyk, S. H. Chen, J. S. Huang, and M. W. Kim, "Structure of three-component microemulsions in the critical region determined by small-angle neutron scattering," Physical Review A, vol. 29, no. 4, pp. 2054-2069, 1984.

[40] V. A. Frolov, A. V. Shnyrova, and J. Zimmerberg, "Lipid polymorphisms and membrane shape," Cold Spring Harbor Perspectives in Biology, vol. 3, no. 11, article a004747, 2011.

[41] R. A. Karjiban, N. S. Shaari, U. V. Gunasakaran, and M. Basri, "A coarse-grained molecular dynamics study of DLPC, DMPC, DPPC, and DSPC mixtures in aqueous solution," Journal of Chemistry, vol. 2013, Article ID 931051, 6 pages, 2013.

[42] S. Jain and F. S. Bates, "On the origins of morphological complexity in block copolymer surfactants," Science, vol. 300, no. 5618, pp. 460-464, 2003.

[43] P. Schurtenberger, "Static and dynamic properties of micelles, microemulsions and vesicles," Current Opinion in Colloid \& Interface Science, vol. 1, no. 6, pp. 773-778, 1996.

[44] S. Buček, S. Kralj, and T. J. Sluckin, "Hysteresis in twodimensional liquid crystal models," Advances in Condensed Matter Physics, vol. 2015, Article ID 834867, 10 pages, 2015.

[45] A. M. Talarico, M. Ghedini, C. O. Rossi, and E. I. Szerb, "Thermotropic iridium(III)-based liquid crystal in amphiphilic environment," Soft Matter, vol. 8, no. 46, pp. 1166111669, 2012.

[46] A. M. Talarico, E. I. Szerb, M. Ghedini, and C. O. Rossi, “The potential of the F127-water soft system towards selective solubilisation of iridium(III) octahedral complexes," Soft Materials, vol. 10, no. 35, pp. 6783-6790, 2014.

[47] M. L. H. Green and G. Parkin, "Application of the covalent bond classification method for the teaching of inorganic chemistry," Journal of Chemical Education, vol. 91, no. 6, pp. 807-816, 2014.

[48] R. G. Pearson, "Recent advances in the concept of hard and soft acids and bases," Journal of Chemical Education, vol. 64, no. 7, pp. 561-567, 1987.

[49] R. J. Lundgren and M. Stradiotto, "Key Concepts in Ligand Design," in An Introduction, chapter 1 in Ligand Design in
Metal Chemistry: Reactivity and Catalysis, M. Stradiotto and R. J. Lundgren, Eds., pp. 1-14, John Wiley \& Sons, Ltd. Published, 2016.

[50] O. M. Yaghi, M. O'Keeffe, N. W. Ockwig, H. K. Chae, M. Eddaoudi, and J. Kim, "Reticular synthesis and the design of new materials," Nature, vol. 423, no. 6941, pp. 705-714, 2003.

[51] A. Nimmermark, L. Ohrstrom, and J. Reedijk, "Metal-ligand bond lengths and strengths: are they correlated? A detailed CSD analysis," Zeitschrift für Kristallographie, vol. 228, no. 7, pp. 311-317, 2013.

[52] S. S. Shaik and P. C. Hiberty, A Chemist's Guide to Valence Bond Theory, Wiley-Interscience, New Jersey, 2008.

[53] I. D. Brown, The Chemical Bond in Inorganic Chemistry: The Bond Valence Model, Oxford University Press, Oxford, New York, USA, 2002.

[54] K. Bian, H. Schunk, D. Ye et al., "Formation of self-assembled gold nanoparticle supercrystals with facet-dependent surface plasmonic coupling," Nature Communications, vol. 9, no. 1, article 2365, 2018.

[55] S. Ortega, M. Ibáñez, Y. Liu et al., "Bottom-up engineering of thermoelectric nanomaterials and devices from solutionprocessed nanoparticle building blocks," Chemical Society Reviews, vol. 46, no. 12, pp. 3510-3528, 2017.

[56] Y. Yin and A. P. Alivisatos, "Colloidal nanocrystal synthesis and the organic-inorganic interface," Nature, vol. 437, no. 7059, pp. 664-670, 2005.

[57] J. Kim, H. . S. Kim, N. Lee et al., "Multifunctional uniform nanoparticles composed of a magnetite nanocrystal core and a mesoporous silica shell for magnetic resonance and fluorescence imaging and for drug delivery," Angewandte Chemie International Edition, vol. 47, no. 44, pp. 84388441, 2008.

[58] J. Kim, J. E. Lee, J. Lee et al., "Magnetic fluorescent delivery vehicle using uniform mesoporous silica spheres embedded with monodisperse magnetic and semiconductor nanocrystals," Journal of the American Chemical Society, vol. 128, no. 3, pp. 688-689, 2006.

[59] W. Wenbo, F. Bai, and H. Fan, "Surfactant-assisted cooperative self-assembly of nanoparticles into active nanostructures," Science, vol. 11, pp. 272-293, 2019.

[60] F. Mallamace, R. Beneduci, P. Gambadauro, D. Lombardo, and S. H. Chen, "Glass and percolation transitions in dense attractive micellar system," Physica A: Statistical Mechanics and its Application, vol. 302, no. 1-4, pp. 202-219, 2001.

[61] P. Calandra, "On the physico-chemical basis of selfnanosegregation giving magnetically-induced birefringence in dibutyl phosphate/bis(2-ethylhexyl) amine systems," Journal of Molecular Liquids, vol. 310, Article ID 113186, 2020.

[62] P. J. Collings, J. N. Goldstein, E. J. Hamilton, B. R. Mercado, K. J. Nieser, and M. H. Regan, "The nature of the assembly process in chromonic liquid crystals," Liquid Crystals Reviews, vol. 3, no. 1, pp. 1-27, 2015.

[63] P. Calandra, C. Giordano, A. Ruggirello, and V. Turco Liveri, "Physicochemical investigation of acrylamide solubilization in sodium bis(2-ethylhexyl)sulfosuccinate and lecithin reversed micelles," Journal of Colloid and Interface Science, vol. 277, no. 1, pp. 206-214, 2004.

[64] D. A. Vanden Bout, "Metal nanoparticle - synthesis, characterizations and applications," Journal of the American Chemical Society, vol. 124, no. 26, pp. 7874-7875, 2002. 
[65] J. Eastoe, M. J. Hollamby, and L. Hudson, "Recent advances in nanoparticle synthesis with reversed micelles," Advances in Colloid and Interface Science, vol. 128-130, pp. 5-15, 2006.

[66] P. Calandra, I. Nicotera, C. O. Rossi, and V. T. Liveri, "Dynamical properties of self-assembled surfactant-based mixtures: triggering of one-dimensional anomalous diffusion in bis(2-ethylhexyl)phosphoric acid/n-octylamine systems," Langmuir, vol. 29, no. 48, pp. 14848-14854, 2013.

[67] L. Bonaccorsi, P. Calandra, M. A. Kiselev, H. Amenitsch, E. Proverbio, and D. Lombardo, "Self-assembly in poly(dimethylsiloxane)-poly (ethylene oxide) block copolymer template directed synthesis of linde type A zeolite," Langmuir, vol. 29, no. 23, pp. 7079-7086, 2013.

[68] L. Bonaccorsi, P. Calandra, H. Amenitsch, E. Proverbio, and D. Lombardo, "Growth of fractal aggregates during template directed SAPO-34 zeolite formation," Microporous and Mesoporous Materials, vol. 167, pp. 3-9, 2013.

[69] K. Rajagopal and J. P. Schneider, "Self-assembling peptides and proteins for nanotechnological applications," Current Opinion in Structural Biology, vol. 14, no. 4, pp. 480-486, 2004.

[70] M. G. Ryadnov and D. N. Woolfson, "Engineering the morphology of a self-assembling protein fibre," Nature Materials, vol. 2, no. 5, pp. 329-332, 2003.

[71] P. Calandra, V. Turco Liveri, P. Riello, I. Freris, and A. Mandanici, "Self-assembly in surfactant-based liquid mixtures: octanoic acid/bis (2-ethylhexyl) amine systems," Journal of Colloid and Interface Science, vol. 367, no. 1, pp. 280-285, 2012.

[72] P. Calandra, V. Turco Liveri, A. M. Ruggirello, M. Licciardi, D. Lombardo, and A. Mandanici, "Anti-Arrhenian behaviour of conductivity in octanoic acid-bis(2-ethylhexyl) amine systems: a physico-chemical study," Journal of Materials Chemistry C, vol. 3, no. 13, pp. 3198-3210, 2015.

[73] P. Calandra, A. Ruggirello, A. Pistone, and V. Turco Liveri, "Structural and optical properties of novel surfactant coated $\mathrm{TiO}_{2}-\mathrm{Ag}$ based nanoparticles," Journal of Cluster Science, vol. 21, no. 4, pp. 767-778, 2010.

[74] A. Petitjean, L. A. Cuccia, J. M. Lehn, H. Nierengarten, and M. Schmutz, "Cation-promoted hierarchical formation of supramolecular assemblies of self-organized helical molecular components," Angewandte Chemie International Edition in English, vol. 41, no. 7, pp. 1195-1198, 2002.

[75] C. K. Gupta and T. K. Mukherjee, Hydrometallurgy in Extraction Processes, Volume II, CRC Press Inc., Boca Raton, Florida USA, 2000.

[76] K. Youshizuka, M. Koba, and K. Inoue, Solvent extraction of rare earth elements with bis (4-ethylcyclohexyl) phosphoric acid, in solvent extraction in the process industries, D. $\mathrm{H}$. Logsdail and M. J. Slater, Eds., Solvent Extraction in the Process Industries, 1993.

[77] N. Zhou, J. Wu, Z. Yu, R. D. Neuman, D. Wang, and G. Xu, "Investigation of aggregation in solvent extraction of lanthanides by acidic extractants (organophosphorus and naphthenic acid)," Science in China Series B-Chemistry, vol. 40, no. 1, pp. 61-71, 1997.

[78] J. Wu, N. Zhou, D. Wang, Z. Yu, R. D. Neuman, and G. Xu, "Structure and aggregation behavior of cobalt-di (2-ethylhexy 1)-phosphoric acid complexes in solvent extraction systems," Science in China Series B-Chemistry, vol. 40, no. 1, pp. 36-44, 1998.
[79] Z. Yuan, Z. Yin, S. Sun, and J. Hao, “Densely stacked multilamellar and oligovesicular vesicles, bilayer cylinders, and tubes joining with vesicles of a salt-free catanionic extractant and surfactant system," The Journal of Physical Chemistry B, vol. 112, no. 5, pp. 1414-1419, 2008.

[80] W. Guo, S. Chen, Y. Feng, and C. Yang, "Investigations of triphenyl phosphate and bis-(2-ethylhexyl) phosphate selfassembled films on iron surface using electrochemical methods, Fourier transform infrared spectroscopy, and molecular simulations," The Journal of Physical Chemistry C, vol. 111, no. 7, pp. 3109-3115, 2007.

[81] P. Calandra, M. Cioni, V. La Parola, and T. de Caro, "Alkylophosphates as low-cost inhibitors in silver tarnishing," Atti dell'accademia peloritana dei pericolanti - classe di scienze fisiche matematiche e naturali, vol. 97, no. S2, p. A13, 2019.

[82] Q. Zhao et al., "Phosphorescent heavy-metal complexes for bioimaging," Chemical Society Reviews, vol. 40, no. 5, pp. 2508-2524, 2011.

[83] X. Ma, X. Shi, S. Bai et al., "Water-soluble fluorescent unimolecular micelles: ultra-small size, tunable fluorescence emission from the visible to NIR region and enhanced biocompatibility for in vitro and in vivo bioimaging," Chemical Communications, vol. 54, no. 49, pp. 6252-6255, 2018.

[84] J. Li, J. Wang, H. Li, N. Song, D. Wang, and B. Z. Tang, "Supramolecular materials based on AIE luminogens (AIEgens): construction and applications," Chemical Society Reviews, vol. 49, no. 4, pp. 1144-1172, 2020.

[85] H. Shi, X. Ma, Q. Zhao et al., "Ultrasmall phosphorescent polymer dots for ratiometric oxygen sensing and photodynamic cancer therapy," Advanced Functional Materials, vol. 24, no. 30, pp. 4823-4830, 2014.

[86] Q. Zhao, X. Zhou, T. Cao et al., "Fluorescent/phosphorescent dual-emissive conjugated polymer dots for hypoxia bioimaging," Chemical Science, vol. 6, no. 3, pp. 1825-1831, 2015.

[87] T. Pan, C. Yang, J. Shi et al., "Dual pH and oxygen luminescent nanoprobes based on graft polymers for extracellular metabolism monitoring and intracellular imaging," Sensors \& Actuators B: Chemical, vol. 291, pp. 306-318, 2019.

[88] M. Mauro, A. Aliprandi, D. Septiadi, N. S. Kehr, and L. de Cola, "When self-assembly meets biology: luminescent platinum complexes for imaging applications," Chemical Society Reviews, vol. 43, no. 12, pp. 4144-4166, 2014.

[89] Y. Fan et al., "Water-soluble triscyclometalated organoiridium complex: phosphorescent nanoparticle formation, nonlinear optics, and application for cell imaging," ACS Applied Materials \& Interfaces, vol. 6, no. 5, pp. 3122-3131, 2014.

[90] R. Talhout and J. B. F. N. Engberts, "Self-assembly in mixtures of sodium alkyl sulfates and alkyltrimethylammonium bromides: aggregation behavior and catalytic properties," Langmuir, vol. 13, no. 19, pp. 5001-5006, 1997.

[91] P.-Y. Ho et al., "Recent advances of iridium (III) metallophosphors for health-related applications," Coordination Chemistry Reviews, vol. 413, p. 213267, 2020.

[92] E. Babu, J. Bhuvaneswari, P. M. Mareeswaran, P. Thanasekaran, H.-M. Lee, and S. Rajagopal, "Transition metal complexes based aptamers as optical diagnostic tools for disease proteins and biomolecules," Coordination Chemistry Reviews, vol. 380, pp. 519-549, 2019.

[93] C. N. Ko, G. Li, C. H. Leung, and D. L. Ma, "Dual function luminescent transition metal complexes for cancer 
theranostics: the combination of diagnosis and therapy," Coordination Chemistry Reviews, vol. 381, pp. 79-103, 2019.

[94] V. W.-W. Yam and A. S.-Y. Law, "Luminescent $d^{8}$ metal complexes of platinum(II) and gold (III): from photophysics to photofunctional materials and probes," Coordination Chemistry Reviews, vol. 414, p. 213298, 2020.

[95] A. C. Sedgwick, J. T. Brewster, P. Harvey et al., "Metal-based imaging agents: progress towards interrogating neurodegenerative disease," Chemical Society Reviews, vol. 49, no. 10, pp. 2886-2915, 2020.

[96] A. Aliprandi, M. Mauro, and L. De Cola, "Controlling and imaging biomimetic self-assembly," Nature Chemistry, vol. 8, no. 1, pp. 10-15, 2016.

[97] M. Mauro, A. Aliprandi, C. Cebrián, D. Wang, C. Kübel, and L. de Cola, "Self-assembly of a neutral platinum(II) complex into highly emitting microcrystalline fibers through metallophilic interactions," Chemical Communications, vol. 50, no. 55, pp. 7269-7272, 2014.

[98] C. A. Strassert, C. H. Chien, M. D. Galvez Lopez et al., "Switching on luminescence by the self-assembly of a platinum(II) complex into gelating nanofibers and electroluminescent films," Angewandte Chemie International Edition, vol. 50, no. 4, pp. 946-950, 2011.

[99] S. Sinn, L. Yang, F. Biedermann et al., “Templated formation of luminescent virus-like particles by tailor-made $\mathrm{Pt}(\mathrm{II})$ amphiphiles," Journal of the American Chemical Society, vol. 140, no. 6, pp. 2355-2362, 2018.

[100] Y. Atoini, E. A. Prasetyanto, P. Chen, S. Silvestrini, J. Harrowfield, and L. de Cola, "Luminescence of amphiphilic PtII complexes controlled by confinement," Chemistry - a European Journal, vol. 24, no. 46, pp. 12054-12060, 2018.

[101] M. E. Robinson, D. J. Lunn, A. Nazemi, G. R. Whittell, L. de Cola, and I. Manners, "Length control of supramolecular polymeric nanofibers based on stacked planar platinum(II) complexes by seeded-growth," Chemical Communications, vol. 51, no. 88, pp. 15921-15924, 2015.

[102] M. E. Robinson, A. Nazemi, D. J. Lunn et al., "Dimensional control and morphological transformations of supramolecular polymeric nanofibers based on cofacially-stacked planar amphiphilic platinum(II) complexes," ACS Nano, vol. 11, no. 9, pp. 9162-9175, 2017.

[103] Y. Li, L. Chen, Y. Ai, E. Y. H. Hong, A. K. W. Chan, and V. W. W. Yam, "Supramolecular self-assembly and dual-switch vapochromic, vapoluminescent, and resistive memory behaviors of amphiphilic platinum(II) complexes," Journal of the American Chemical Society, vol. 139, no. 39, pp. 1385813866, 2017.

[104] C. Po, A. Y. Y. Tam, K. M. C. Wong, and V. W. W. Yam, "Supramolecular self-assembly of amphiphilic anionic platinum(II) complexes: a correlation between spectroscopic and morphological properties," Journal of the American Chemical Society, vol. 133, no. 31, pp. 12136-12143, 2011.

[105] C. Po, A. Y. Y. Tam, and V. W. W. Yam, "Tuning of spectroscopic properties via variation of the alkyl chain length: a systematic study of molecular structural changes on selfassembly of amphiphilic sulfonate-pendant platinum(II) bzimpy complexes in aqueous medium," Chemical Science, vol. 5, no. 7, pp. 2688-2695, 2014.

[106] C. Po and V. W.-W. Yam, "A metallo-amphiphile with unusual memory behaviour: effect of temperature and structure on the self-assembly of triethylene glycol (TEG)-pen- dant platinum(II) bzimpy complexes," Chemical Science, vol. 5, no. 12, pp. 4868-4872, 2014.

[107] N. Liu, B. Wang, W. Liu, and W. Bu, "Reversible luminescence switching accompanied by assembly-disassembly of metallosupramolecular amphiphiles based on a platinum(II) complex," Journal of Materials Chemistry C, vol. 1, no. 6, pp. 1130-1136, 2013.

[108] M. H. Y. Chan, S. Y. L. Leung, and V. W. W. Yam, “Controlling self-assembly mechanisms through rational molecular design in oligo(p-phenyleneethynylene)-containing alkynylplatinum(II) 2,6-bis(N-alkylbenzimidazol-2' -yl)pyridine amphiphiles," Journal of the American Chemical Society, vol. 140, no. 24, pp. 7637-7646, 2018.

[109] F. Camerel, R. Ziessel, B. Donnio et al., "Formation of gels and liquid crystals induced by Pt $\cdots \mathrm{Pt}$ and $\pi-\pi *$ interactions in luminescent $\sigma$-alkynyl platinum(II) terpyridine complexes," Angewandte Chemie International Edition, vol. 46, no. 15, pp. 2659-2662, 2007.

[110] A. Y. Y. Tam, K. M. C. Wong, G. Wang, and V. W. W. Yam, "Luminescent metallogels of platinum(II) terpyridyl complexes: interplay of metal...metal, $\pi-\pi$ and hydrophobichydrophobic interactions on gel formation," Chemical Communications, vol. 28, no. 20, pp. 2028-2030, 2007.

[111] A. Y. Y. Tam and V. W. W. Yam, "Recent advances in metallogels," Chemical Society Reviews, vol. 42, no. 4, pp. 15401567, 2013.

[112] C. Yu, K. . H. Y. Chan, K. . M. C. Wong, and V. . W. W. Yam, "Polyelectrolyte-induced self-assembly of positively charged alkynylplatinum(II)-terpyridyl complexes in aqueous media," Chemistry-a European Journal, vol. 14, no. 15, pp. 4577-4584, 2008.

[113] H. L. K. Fu, C. Po, S. Y. L. Leung, and V. W. W. Yam, "Selfassembled architectures of alkynylplatinum(II) amphiphiles and their structural optimization: a balance of the interplay among Pt $\cdots \mathrm{Pt}, \pi-\pi$ stacking, and hydrophobic-hydrophobic interactions," ACS Applied Materials \& Interfaces, vol. 9, no. 3, pp. 2786-2795, 2017.

[114] Y. Wang, Y. Cai, L. Cao et al., "An amphiphilic metallaclip with enhanced fluorescence emission in water: synthesis and controllable self-assembly into multidimensional micro-structures," Chemical Communications, vol. 55, no. 68, pp. 10132-10134, 2019.

[115] Y. Wang, C. Wang, R. Long et al., "Synthesis and controllable self-assembly of 3D amphiphilic organoplatinum(II) metallacages in water," Chemical Communications, vol. 55, no. 35, pp. 5167-5170, 2019.

[116] C. Cebrián, M. Natali, D. Villa et al., "Luminescent supramolecular soft nanostructures from amphiphilic dinuclear $\operatorname{Re}(\mathrm{I})$ complexes," Nanoscale, vol. 7, no. 28, pp. 12000-12009, 2015.

[117] B. Manimaran, P. Thanasekaran, T. Rajendran et al., "Luminescence enhancement induced by aggregation of alkoxybridged rhenium(I) molecular rectangles," Inorganic Chemistry, vol. 41, no. 21, pp. 5323-5325, 2002.

[118] A. Guerrero-Martínez, Y. Vida, D. Domínguez-Gutiérrez, R. Q. Albuquerque, and L. De Cola, "Tuning emission properties of iridium and ruthenium metallosurfactants in micellar systems," Inorganic Chemistry, vol. 47, no. 20, pp. 9131-9133, 2008.

[119] M. Mauro, G. de Paoli, M. Otter, D. Donghi, G. D'Alfonso, and L. de Cola, "Aggregation induced colour change for phosphorescent iridium(III) complex-based anionic 
surfactants," Dalton Transactions, vol. 40, no. 45, pp. 1210612116, 2011.

[120] B. Kemper, Y. R. Hristova, S. Tacke et al., "Facile synthesis of a peptidic $\mathrm{Au}(\mathrm{I})$-metalloamphiphile and its self-assembly into luminescent micelles in water," Chemical Communications, vol. 51, no. 25, pp. 5253-5256, 2015.

[121] A. Masters, "Chromonic liquid crystals: more questions than answers," Liquid Crystal Today, vol. 25, no. 2, pp. 30-37, 2016.

[122] P. J. Collings, A. J. Dickinson, and E. C. Smith, "Molecular aggregation and chromonic liquid crystals," Liquid Crystals, vol. 37, no. 6-7, pp. 701-710, 2010.

[123] Q. Gao, C. Zou, and W. Lu, "Lyotropic chromonic mesophases derived from metal-organic complexes," Chemistry: An Asian Journal, vol. 13, no. 21, pp. 3092-3105, 2018.

[124] X. S. Xiao, C. Zou, X. Guan, C. Yang, W. Lu, and C. M. Che, "Homoleptic gold(I) N-heterocyclic allenylidene complexes: excited-state properties and lyotropic chromonics," Chemical Communications, vol. 52, no. 28, pp. 4983-4986, 2016.

[125] D. Pucci, B. S. Mendiguchia, C. M. Tone et al., "Unconventionally shaped chromonic liquid crystals formed by novel silver(I) complexes," Journal of Materials Chemistry C, vol. 2, no. 41, pp. 8780-8788, 2014.

[126] W. Lu, Y. Chen, V. . A. . L. Roy, S. . S. Y. Chui, and C. M. Che, "Supramolecular polymers and chromonic mesophases selforganized from phosphorescent cationic organoplatinum(II) complexes in water," Angewandte Chemie International Edition, vol. 48, no. 41, pp. 7621-7625, 2009.

[127] X. S. Xiao, W. Lu, and C. M. Che, "Phosphorescent nematic hydrogels and chromonic mesophases driven by intra- and intermolecular interactions of bridged dinuclear cyclometalated platinum(II) complexes," Chemical Science, vol. 5, no. 6, pp. 2482-2488, 2014.

[128] Y. Chen, K. Li, H. O. Lloyd, W. Lu, S. S. Y. Chui, and C. M. Che, "Tetrakis(arylisocyanide) rhodium(I) salts in water: NIR luminescent and conductive supramolecular polymeric nanowires with hierarchical organization," Angewandte Chemie International Edition, vol. 49, no. 51, pp. 9968-9971, 2010.

[129] Y. J. Yadav, B. Heinrich, G. De Luca et al., "Chromonic-like physical luminescent gels formed by ionic octahedral iridium(III) complexes in diluted water solutions," Advanced Optical Materials, vol. 1, no. 11, pp. 844-854, 2013.

[130] A. F. Henwood and E. Zysman-Colman, "Luminescent iridium complexes used in light-emitting electrochemical cells (LEECs)," Topics in Current Chemistry, vol. 374, no. 4, 2016.

[131] R. D. Costa, E. Ortí, H. J. Bolink et al., “Archetype cationic iridium complexes and their use in solid-state light-emitting electrochemical cells," Advanced Functional Materials, vol. 19, no. 21, pp. 3456-3463, 2009.

[132] C. O. Rossi, C. Cretu, L. Ricciardi et al., "Rheological and photophysical investigations of chromonic-like supramolecular mesophases formed by luminescent iridium(III) ionic complexes in water," Liquid Crystals, vol. 44, no. 5, pp. 880-888, 2017.

[133] H. B. Bohidar and K. Rawat, Design of Nanostructures: SelfAssembly of Nanomaterials, Wiley, 2017.

[134] M. Pochylski, D. Lombardo, and P. Calandra, "Optical birefringence growth driven by magnetic field in liquids: the case of dibutyl phosphate/propylamine system," Applied Sciences, vol. 10, no. 1, p. 164, 2020.
[135] H. Wakayama and H. Yonekura, "Synthesis of inorganic nanocomposites by selective introduction of metal complexes into a self-assembled block copolymer template," Journal of Nanomaterials, vol. 2015, Article ID 905083, 6 pages, 2015.

[136] P. Calandra, T. de Caro, D. Caschera, D. Lombardo, L. Todaro, and V. Turco Liveri, "Spectroscopic and structural characterization of pure and $\mathrm{FeCl} 3$-containing tri-n-butyl phosphate," Colloid and Polymer Science, vol. 293, no. 2, pp. 597-603, 2015.

[137] K. Ariga, M. Nishikawa, T. Mori, J. Takeya, L. K. Shrestha, and J. P. Hill, "Self-assembly as a key player for materials nanoarchitectonics," Science and Technology of Advanced Materials, vol. 20, no. 1, pp. 51-95, 2019.

[138] W. Kong, C. Chen, K. Mai, X. Shi, R. Hu, and Z. Wang, "Large-scale synthesis and self-assembly of monodisperse spherical $\mathrm{TiO}_{2}$ Nanocrystals," Journal of Nanomaterials, vol. 2011, Article ID 526246, 4 pages, 2011.

[139] P. Calandra, D. Lombardo, A. Pistone, V. Turco Liveri, and S. Trusso, "Structural and optical properties of novel surfactant-coated $\mathrm{Yb} @ \mathrm{TiO}_{2}$ nanoparticles," Journal of Nanoparticle Research, vol. 13, no. 11, pp. 5833-5839, 2011.

[140] B. A. Grzybowski and W. T. S. Huck, "The nanotechnology of life-inspired systems," Nature Nanotechnology, vol. 11, no. 7, pp. 585-592, 2016.

[141] W.-J. Kim, J. T. Seo, C. S. Ah et al., "Colorimetric analysis on flocculation of bioinspired $\mathrm{Au}$ self-assembly for biophotonic application," Journal of Nanomaterials, vol. 2009, Article ID 261261, 6 pages, 2009.

[142] D. Lombardo, P. Calandra, D. Barreca, S. Magazù, and M. Kiselev, "Soft interaction in liposome nanocarriers for therapeutic drug delivery," Nanomaterials, vol. 6, no. 7, p. $125,2016$.

[143] D. Lombardo, M. A. Kiselev, S. Magazù, and P. Calandra, "Amphiphiles self-assembly: basic concepts and future perspectives of supramolecular approaches," Advances in Condensed Matter Physics, vol. 2015, Article ID 151683, 22 pages, 2015.

[144] T. Jiao, R. Xing, Q. Zhang, Y. Lv, J. Zhou, and F. Gao, "Selfassembly, interfacial nanostructure, and supramolecular chirality of the Langmuir-Blodgett films of some Schiff base derivatives without alkyl chain," Journal of Nanomaterials, vol. 2013, Article ID 297564, 9 pages, 2013.

[145] K. R. Berry, R. L. Romo, M. Mitchell, V. Bejugam, and D. K. Roper, "Controlled gold nanoparticle placement into patterned polydimethylsiloxane thin films via directed selfassembly," Journal of Nanomaterials, vol. 2019, Article ID 5390562, 11 pages, 2019. 